RACAR : Revue d'art canadienne

Canadian Art Review

RACAR

\title{
La rose de Notre-Dame de Noyon et sa place dans la technique et le décor du troisième chantier de la cathédrale
}

\section{Chantal Hardy}

Volume 13, numéro 1, 1986

URI : https://id.erudit.org/iderudit/1073554ar

DOI : https://doi.org/10.7202/1073554ar

Aller au sommaire du numéro

Éditeur(s)

UAAC-AAUC (University Art Association of Canada | Association d'art des universités du Canada)

ISSN

0315-9906 (imprimé)

1918-4778 (numérique)

Découvrir la revue

Citer cet article

Hardy, C. (1986). La rose de Notre-Dame de Noyon et sa place dans la technique et le décor du troisième chantier de la cathédrale. RACAR : Revue d'art canadienne / Canadian Art Review, 13(1), 7-22. https://doi.org/10.7202/1073554ar

\section{Résumé de l'article}

The rose-window has been very important in the development of fenestrage major elements in Gothic architecture. The earliest examples appear in the middle of the twelfth century on the Île-de-France and are not well-known, particularly those with free-standing colonnettes, a type which Viollet-le-Duc does not include in what is still the basic work of reference for the rose-window. There is a very old window of this type preserved in the cathedral of Noyon. A careful study reveals the importance of the form within the third campaign of the cathedral, ca. 1170-1185. After establishing its authenticity and general date, a detailed analysis places the window within the building, from the point of view of both technique and decoration. Three types of circular window were constructed during the course of this campaign. The rose-window of the Treasury shows the same type of insertion under the vault as the small polylobed oculus at the lower side, north of the nave (1170). The tracery of the rose-window is made of stone slabs cut into cusped voussoirs and of colonnettes en délit, both elements found from the beginning to the end of the campaign. The Treasury rose-window has a rare feature: a primitive method for securing the frame. The armature is fastened to the interior surface of the curved wedges leaving the colonnettes of the tracery free. This system seems to explain the presence of bilobed arches at the border of the frame, an innovation for the rose-window with colonnettes. The decoration of the rose-window is linked to the secondary decoration of the portal of the annex of the north arm of the transept, under the rose-window, as well as to the very ornate high part of the choir. The execution of the rose-window can be dated between these points, ca. 1170-1175. The archivolt of the rose-window includes the mascaron, a very important motif in the decoration of the cathedral of Noyon and also associated with the earliest rose-windows (Saint-Denis, ca. 1140). The workshop of Noyon is an important center in the development of Gothic architecture, particularly for the treatment of its windows grouped by pairs in the double walls system of the transept or composed with an oculus over twin lancets in the Bishop's chapel. This last formula is recognized as the most direct source for the composition of the high windows of the nave of Chartres cathedral. However at Chartres the rose-window hollows out the entire wall beneath the formeret or the vault, often considered an original creation of the Master of Chartres. This type of piercing, however, was also used in the workshop of Noyon for the rose-window of the Treasury. The study of that rose-window accentuates, through this fact, the importance of the workshop in the development of the large elaborate gothic window.
Tous droits réservés @ C UAAC-AAUC (University Art Association of Canada | Association d'art des universités du Canada), 1986
Ce document est protégé par la loi sur le droit d'auteur. L’utilisation des services d’Érudit (y compris la reproduction) est assujettie à sa politique d'utilisation que vous pouvez consulter en ligne.

https://apropos.erudit.org/fr/usagers/politique-dutilisation/ 


\title{
La rose de Notre-Dame de Noyon et sa place dans la technique et le décor du troisième chantier de la cathédrale
}

\author{
C.HAN'TAL HARDY
}

Universsilé de Montréal

L'importance des fenêtres dans le développement de l'église gothique française est recommuc depuis longtemps'. Conc architecture tendra au cours de son développement à évider tout le mur contre les éléments porteurs des roûtes afin de dispenser la lumière colorée de ses vitraux. Ceux-ci matćrialisaient l'idcé que l'église par ses cloisons translucides. était à l'image de la Jérusalem céleste décrite par l'A pocalypse. la ville céleste aux murs de picrreries". Dans l'enscmble des fenêtres gothiques, les roses présentent un intérêt majeur. Flles sont unc des pièces maîtresses des facades des cathédrales. Do plus, c'est en les associant aux autres fenêtres que les architectes des cathédrales parviendront à ouvirir les murs des travées sous les voûtes des nefs et à crécr le vóritable remplage gothique.

On a peu ćludié l'origince el le développement technique et strlistique de ces immonses réscaux de fenestrages. Lne telle ćtude est difficile car de nombreux problemes d'authenticité se posent relativement aux fenctres : leur entretien au cours des siceles et les restaurations générales du XIX siècle ont très souvent alteré les dispositions d'origine. (Cela est particulicrement vrai pour les fenctres circulaires où furent ćlaborés les premiers châssis de pierre'. L.e groupe des premières roses gothiques est representé par la célèbre rosc-roue de fortune de l'église SaintEtienne de Bcamais, construite vers $1150^{\circ}$. mais ce groupe est mal défini ar les exemples con sont peu nombreux.

I a cathédrale de Voron conserve une rose très peu connuc qui apparticnt à ce groupe (Fig. 1). Apres avoir établi son authenticité nous en ferons l'étude détaillée, de manière à analyser la structure de ce premier modicle de rose à colonnettes libres. Cenc analyse portera sur la technique de construction et sur le décor de la rose en fonction du chanticr de la cathédrale ct, à certains égards, en relation arec l'art de lîle-de-France.
La rose de la cathédrale de Noyon éclaire la salle du trésor, salle d'élage du petit édifice annexe au bras nord du transept. Flle est située à la lave est de l'édifice, qui constituait au XIr" siècle l'unc des deux façades dentrée de la cathédrale (Fig. 2). Ia rose participe au clécor de cette entrée mais clle ne surmonte pas clirectement le portail comme c'est habituellement le cas. Sa position est décalée diunc travéc. ce qui l'éloigne un peu du chevet tout en la rendant beaucoup plus visible à la partie orientale. Sa présence scmble marquer le caractère particulier de la salle du trésor

* Cous remercions virement Mme Delberefue, présidente de la Socićté historieque de Voyon ainsi que M1. L'an (ilabete. archiprêre de la canhedrale de Noron. qui nous ont permis daccéder a la salle dutrésor à lautomne 1 g̣ 1 . Nous remercions également Mme Marie-Claude Béthune pour sessen-

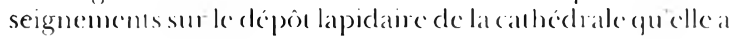

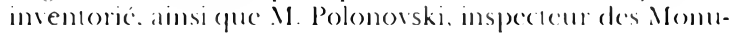
ments historiques. qui nous a permis div avoir alcess (elle recherche a latil lobje diunc communication lors de la deuxieme Canadian (onference of Medieval Mr Mlisto-

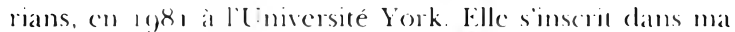

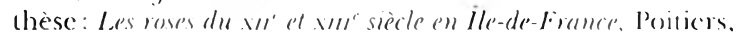
1983

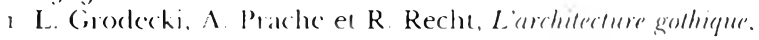
Paris, 197(9) p. 17. ()11 y résume limportance du hiome dans la délinision du strle gothique

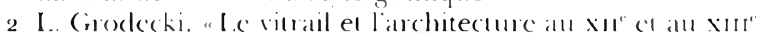

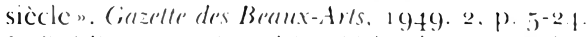

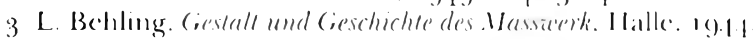

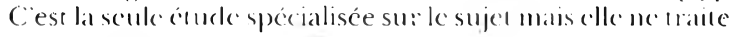
pas de la gencisc de la lenêtre composéc. Violle-te-1)uc

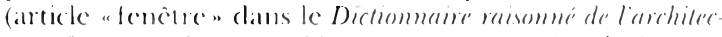

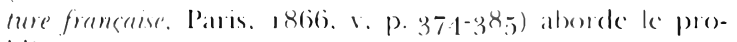
blènc.

4 Notre comnatissance des roses reste encore anjourd hui basée sur l'ótucle de Viollet-le-Duc (lans son Dertiommaire au tome $x$. L a anchitecte ria pas étudié la rose a colomnettes libies qui constituc ke plus ancien modele de rose.

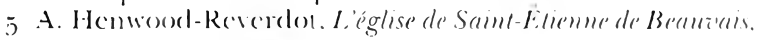

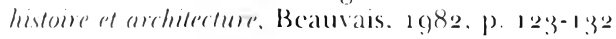




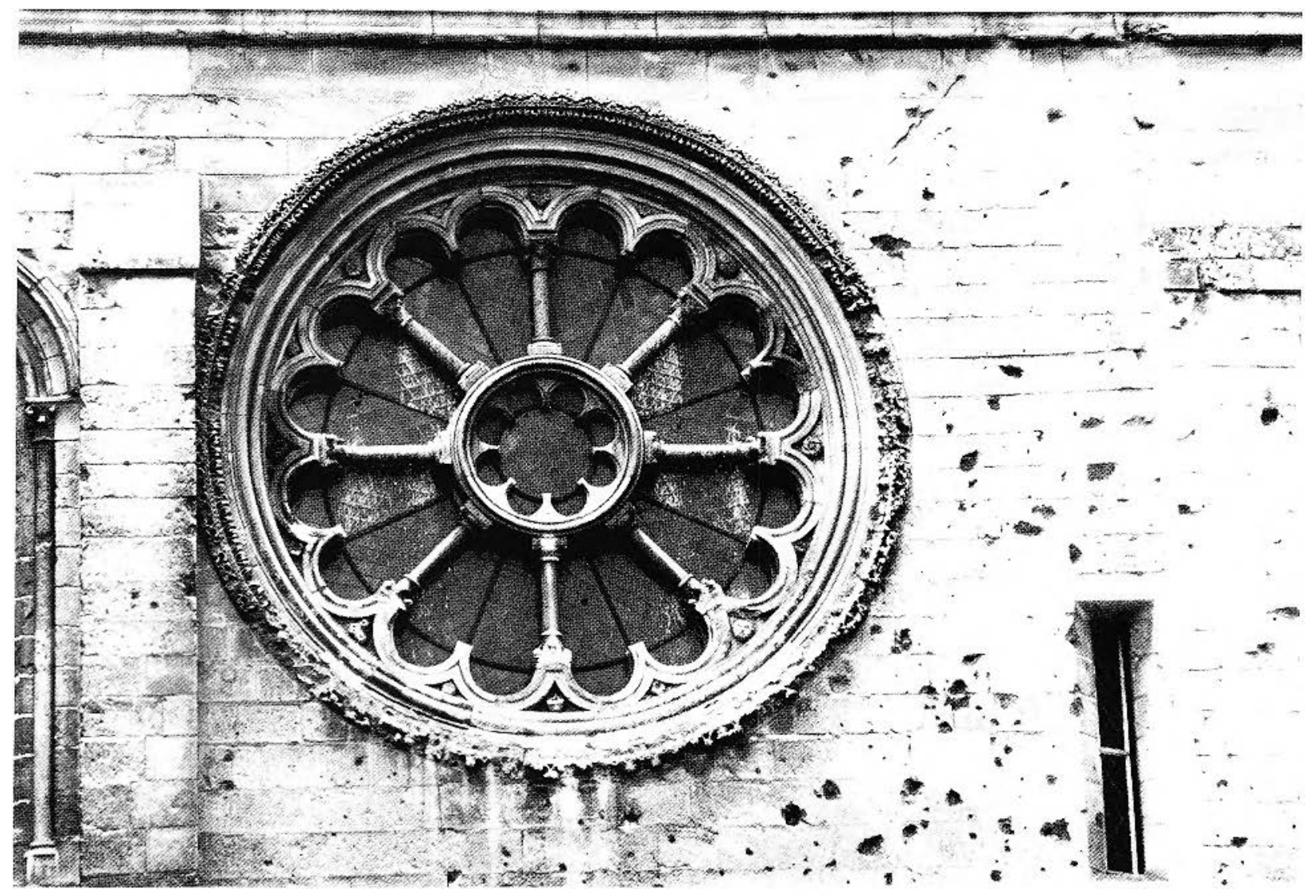

figurf. 1. Cathédrale de Yoýon, rose de la salle du trésor (Photo: Auleur).

La rose de Noyon est une fenêtre circulairc d'environ 3 mètres 40 de diamètre ${ }^{6}$ dont l'encadrement est orné, tant du côté intéricur qu'extérieur, d'un large profil de cordons toriques el de cavets dégagés sur l'axe oblique de l'ébrascment (Fig. 3). A l'extérieur, ce cadre est circonscrit par une archivolte qui lait saillie sur le mur; son décor présente une frise de feuillage d'où émergenı des mascarons. Le châssis de

6 Les dimensions de la rose sont approximatives. Cl. V'. Barbaran. Litude sur l'église de. Noyon et ses déperndances au commurncement du xa' siècle, s.1. 12 dessins non paginés. 1872. I inc copie phorographiée ess comservéc à la bibliothèque de la Direction du patrimoine, ministère de la Culture. Partis. Selon le relevé de Barbaran la rose présente un vide de $3 \mathrm{~m} 4^{(0)}$ son cliamètre, arec le cadre de la baie, alleint $4 \mathrm{~m} 4^{\circ}$.

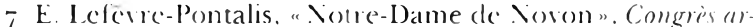
chéologique de France. 190.5. p. 96: "Au-dessus du porche s'ourre une rose du XII" siecle à 10 redents cerclée diun cordon de feuillage. Huit rasons partent de son oculus central at huit lobes.

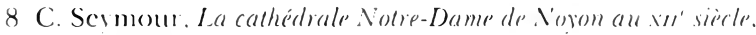

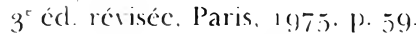

9 "In both treasury and chapel the archited shows himsell u be well alcpuanted with the very latcs developments in window design, the rose paltern in the locasury oculus is comparable to the transept window at Mons-on-Lamonois and the choir tribune windows at St-liticme at Gaten" (Noyon Cathedral. I.cichester, Courtauld Institute Illustrattion Archives. 1983). pierre de la rose est composć d'un cercle périphérique de seize dalles clavées et redentées. I.cs redents plus larges reçoivent les chapitcaux des huit colonnclles posées à partir de l'axc vertical. Celles-ci maintiennent un oeil central constituć de huit dalles égalemenı clavées el redentées. Le remplage de la lenêtre est orné d’un abondant décor de moulures et de feuillages. Celte exécution très soignée fail cle la rose un ćlćment typicjuc du chanticr de Novon, particulièrement réputé pour ses innovations techniques et son décor riche et varić.

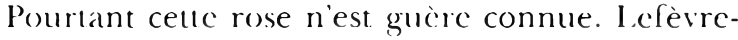
Pontalis la décrit sommairement en $1905^{-}$. Sevmour. en 1939, tout en lui recommaissant quclquc valeur historique, la mentionne en indiquant surtout qu'elle a été complètcment reconstruitc au xrx sièclex. Dans lointroduction au volume de photographies de la cathédrale publić récemment par l'Institut Courtauld. le tracé de celte rose esı comparé à ccux de la rose de Mons-en-laonnois (Fig. 4) et des roses du chrour de Saint-Etienne de (aacni".

Pour nous, la rose de Novon est bien antérieure à ces autres roses. De plus, cllc est parvenuc presque intacte jusquau xix siècle. Elle lut sobremcnt restauréc unc première fois par l'architccte Verdier. puis refaite, entièrcment à l'identiquc, après la première guerre mondiale. C'est ce que nous allons tenter de démontrer. 


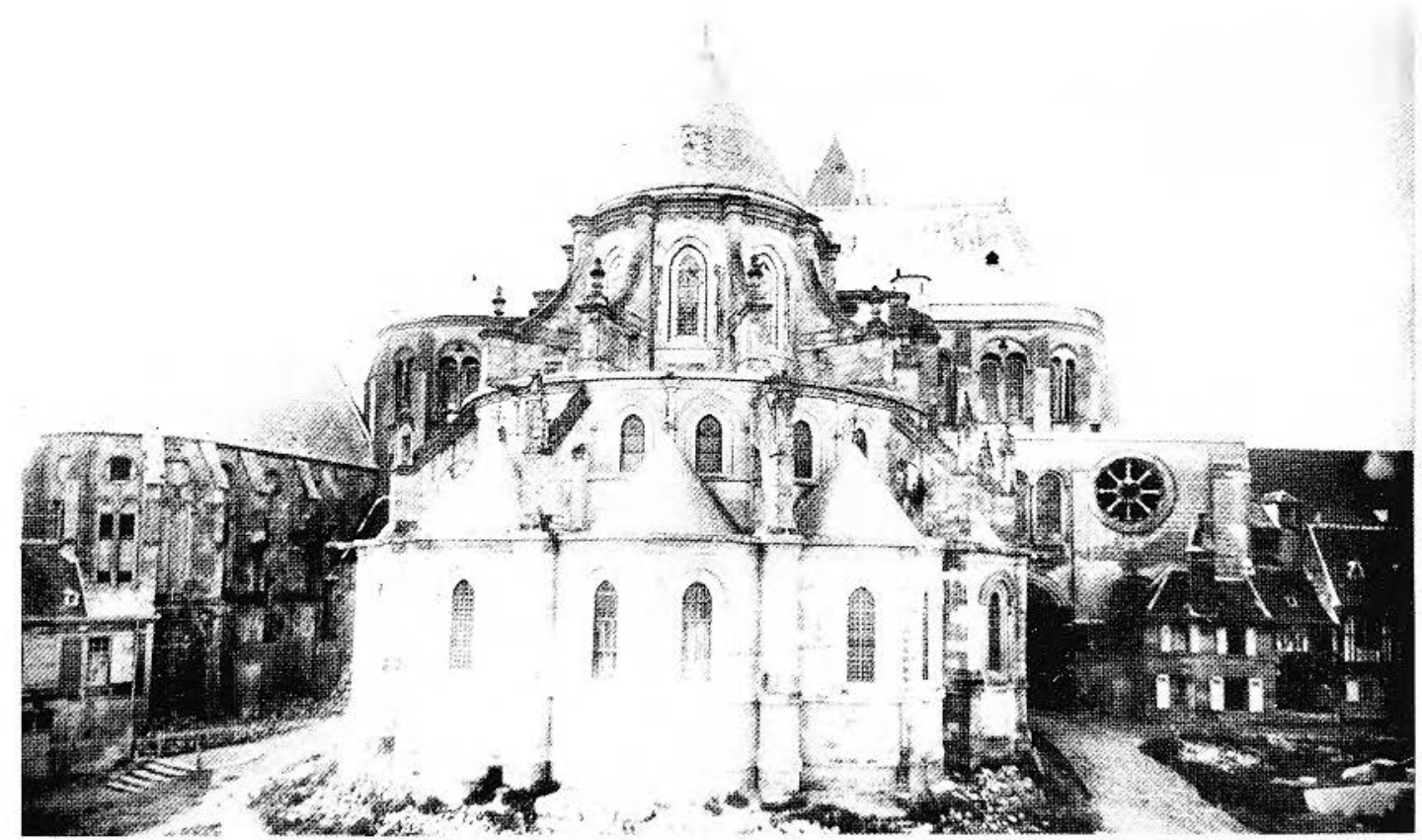

Figure 2. Cathédrale de Noyon, le chevet avec l'annexe au bras nord du transept, avant 1862 (Photo: Arch. Mon. Hist.).
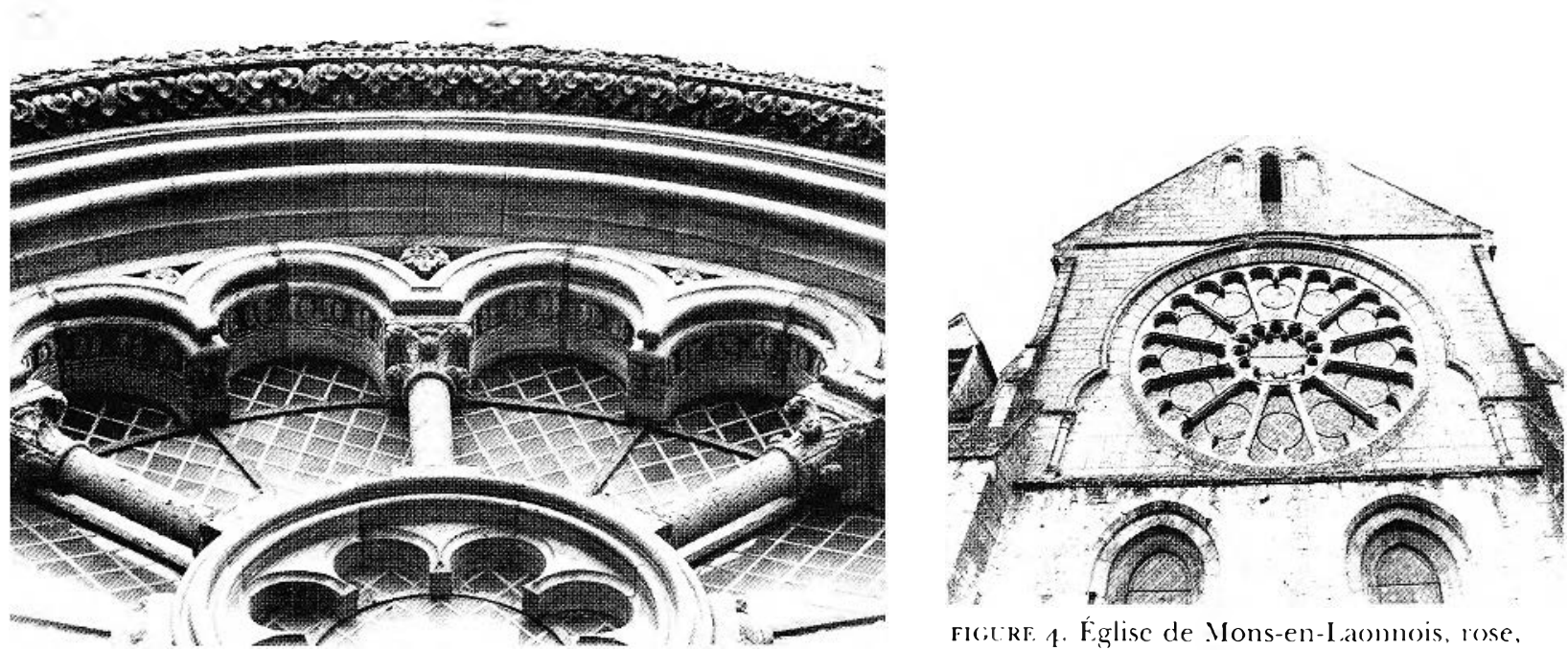

FIGt:RF. 4. Feglise de .Mons-en-Laomois, rose. figure 3. Cathédrale de Noyon, rose du trésor, détail (Photo: Auteur). rers izzo) (Plototo: Auteur).

On comnaît l’étal de la rose arant sà restaumation au xix" siècle par unc gravure publiée en $184.3^{1 "}$ e illustrant le chever de la cathédrale. La salle du wésory apparât privéce de sa toiture: la double fenêtre adjacente à la rose est bouchée, mais celle-ci cst coll bon état et il ne lui manque qu'une colonncle. lin i 8,50 , l'architecte Verdier mentionne la rose dans son projet général de restatuation". La cathédrale est alors privéc de ses vituaux et laisse l'eatu saccumuler à lintérieur de la salle du trésor. Verdier précise dans un devis ulcórieur la mature des travaux a exćculcr: le remplacement de quatre colomettes el de l'orne-

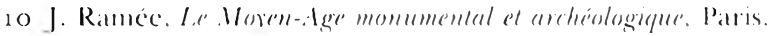

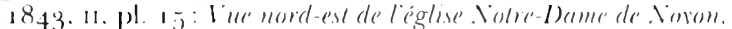
d'après un dessm de Benoist.

1 Projer de restatration générale d Arman Verdier. I)eris de 1850. Parris. Arehieses de conservation des mentuments hisuriques, dessice Oise. 11" 988 . Voron, cathedrale: "An deuxieme porche se trouvelancien trésor duchapitre. cest une grande salle rectangulaire ... éclairéce par une magnifique rosace anjomalhui privée de ses vintaux ... lorsque le

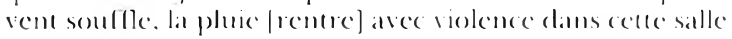

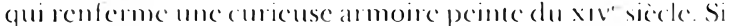
fon ne gannil pas cette rosace de vitratux. ce mouble se détériorerat completement ... Celte salle est reomente de bitume, il fauchat la recourrir de plombs., 
ment dupourtour sur sept mètres. Il propose ćgalement de fermer la baic aree des vilaux blancs:- I ce rappont de linspectem Bocswillwald, daté de 1873. ne lait que montiommor l'achèrement destravaux à la rose. a lexception de la pose des remiores's. ()n no saturait donc conclure, comme Sevmom a pu le penser, que la rose a été contierement relatite" Ce rappont ne précise nila date d'exécution desmavaux nil'éten-

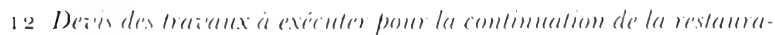
lion. rers 18,7.: "Pour le llansept nesel ... reparation de la

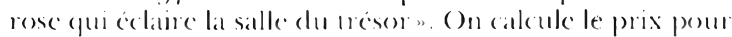

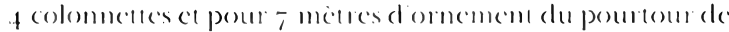

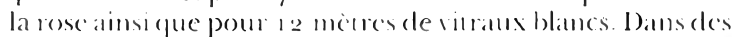

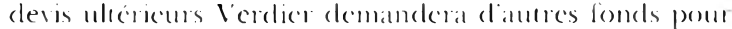

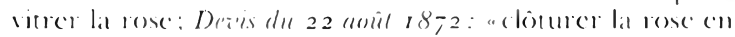

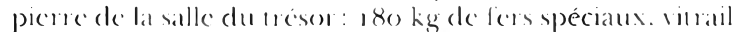
de la rose ('n for domble monté sur plomb) rond (Xiomom.

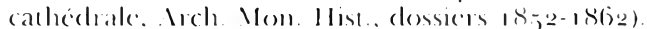

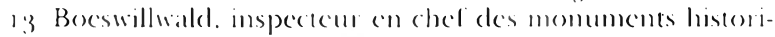

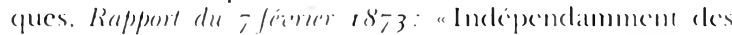

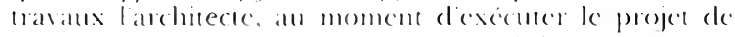

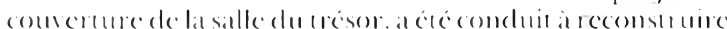

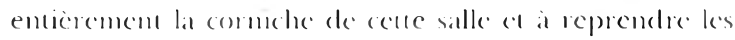
pallements degrades du dessous: ... puis profinam des

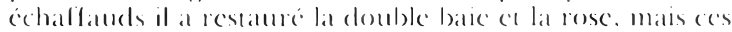

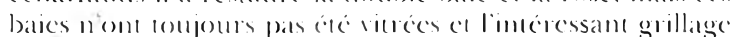

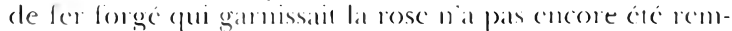
placen (Novom. Cathedralle. Areh. Mom. Mlist.)

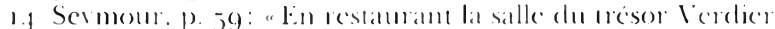

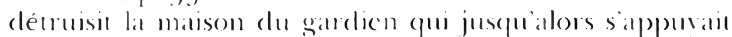

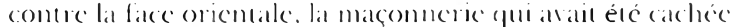

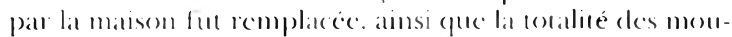

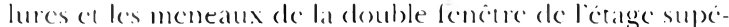

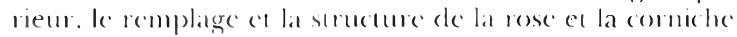

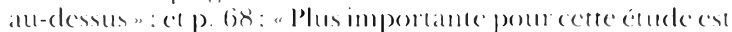

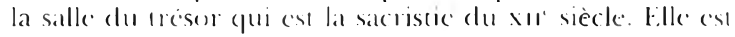

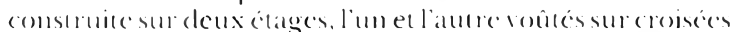

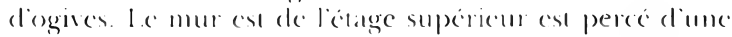

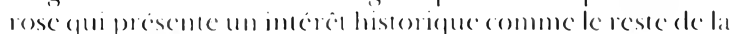
salle, bien (fuclle ait sub) (limportamles restaturatioms."

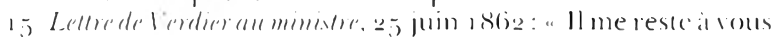

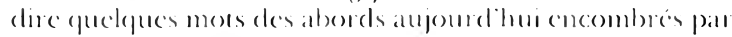
les logements des differents servitems de linglise all grand

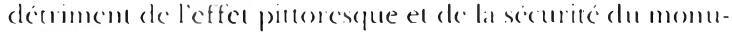

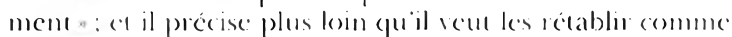

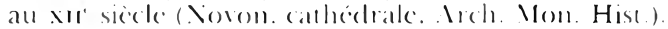

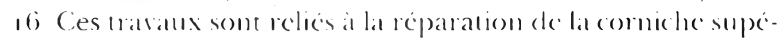

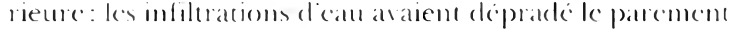

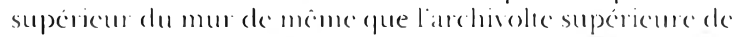

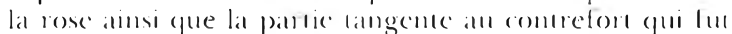
enticerement refatit.

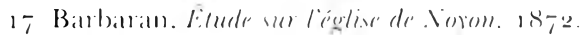

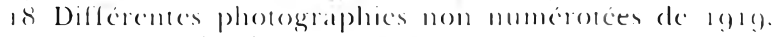

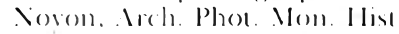

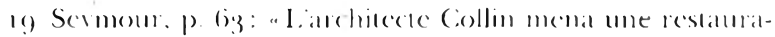

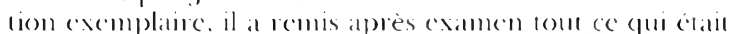
ucilisable, il a remis a lour ('mp)lacement originel les dilli-

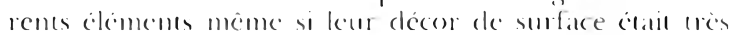

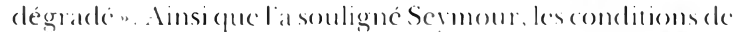

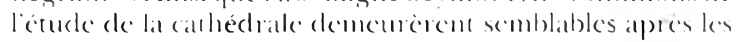

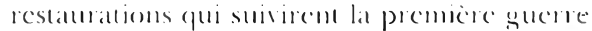

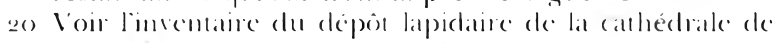

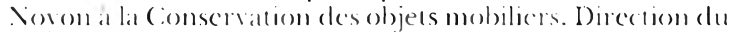

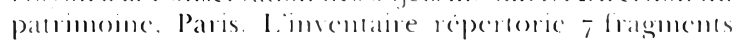

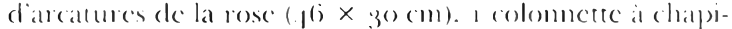

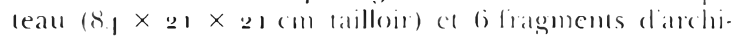

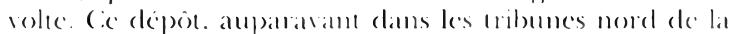
nel. est anjourd hui entrepose dams le grenter de la matison

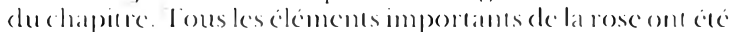

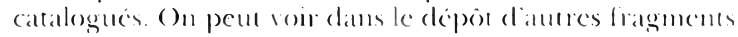

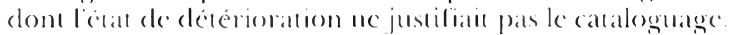

duc de la restauration. alors guc des photographies anciennes, conservés aux archives des momuments historiques, permenemt de pallicr ces lacumes.

()n arait comstruit contre l'amnexe du trésor une maison utiliséc pour loger le gardien, déunite après $1862^{1 .}$. ()r. elle apparait sur une photographie du cherel de la calhódrale (Fig. 2) où nous voyons, déjà restaurécs, la rose, la double fonêtre adjacente et la corniche supéricure de l'ammexe, toujours privée de son toit. La restantration de la rose a donc précédé

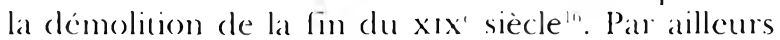
la couleur contrastante de la mone piene plans le réscau de la rose nous perme de juger de l'éendue des reparations el confirme la justesse de l'estimation de licrdier: le rescaut reste on grande partic ancien. um seul clarcau do l'oxil a éré romplacé cl loutes les dalles périphériques ont élé conscrvées. I.es colonnetres sont en grande partic relates. Quant au cordon qui cercle la rose, la partic supérieure a le côté gatuche. contigu alu contrefort. sont neuls. mais la partic a droite et en bas reste intate. Ainsi. cotte vue anciennc et les archives de conservation dómontrent quime grande partie du remplage ancien ful comscrvé lors de la restauration an xix" siècle et que les élements remplacés durent respecter les dispositions d'origine. () n les retrouve d'ailleurs sur le relevé de la rose exéculc par Banbaran vers $1870^{\prime \prime}$. Très précis, du moins en ce qui concerne la structure de la rose a le profil des moulures.

la première guerre mondiale ruina l'édilice, et la rose subit une deuxiome restatuation majeure. (On peur roir sur les photographies de l’éponuc l'état désastreux de son remplage colaté : seuls lés redents périphériques sont restés en place, leur surface est détériorée tout (omme colle du cordon autour de la rose. 1)es photographlies antéricureset postéricures à celle seconde restatuation montrent nettement que la partic inféricure du corclon darchivolte ainsi quiunsegment rersla droite sont d’origine. Ilsont été conscrós malgré leur déćrionations. (2uant au châssis il a été entièrement refait en reproduisant de facon exacte les dispositions ancoriemres, an l'archilecte Collin, qui mena une reconstruction exemplaire de la cathédrale, arait à sa disposition le relevé de Barbaran el différents ćléments de la rosel". Il déposa les élémonts non uriliscs: sept liagments d'arallures el unc colomnelle, de même que quelguces claveaux du cordon d'archivolte $e^{201}$.

Nous sommes donc assurés que la rose actuclle est tout a fait conforme dans sa structure el clans son décor at modìle dorigine. ()n peut en cumdicr les éléments ancicns dóposés do même que quelques clarcaux de la frisc d'archirola restés sur place. Seuls les colommettes el chaphiteaux oflient moins de cortiunde: si l'on se fie i la couleur contrastante du matériau visible sur la pholographic d arant $18($. (lig. 2), unc scule colomncuc, au bas dims l'axe, a. peut-être. été rémployéc par Verdier. Néammoins les colonnetres actuelles reproduisent colles du xix siecle et il restc fort probable quelles furcont exéculces d'après les formes origimales. 
I a clatation globale de la rose pose moins de dillicultés ct se justifie assez bien. T'outcfois. il faudra de plus amples considerations, intéressantes en cllesmêmes, sur les techniques et sur le décor de la rose pour la situer plus précisément dams lévolution du chanticr de la cathédrale. Dòs la fin du sic̀cle dernicr, Lefère-Pontalis attribuat la construction de la salle du trésor à l’épiscopat de Beaudoin $11(1167-1174)$ ed estimait la rose comme dı XII" sic̀cle ${ }^{21}$. I es motices de l'Inventaire du dépôt lapidaire attribucont l'onscmble des éléments de la rose a la lin du xir siècle. ()uant à Sermour il ne date pas la rose de ficen précise. mais en déterminant la chronologic relative des diflérentes partics de la cathédrale mises en service en 118 . il assigne implicitement a la rose une position dans le déroulement des chanticrs"-.

Résumons brièvement ses conclusions qui font maintenant autorité. Le plan du cherel ful fixé lors d'une premiore campagne vers 1150 : c'est à ce moment que les fondations furent jetces ct que les chapclles rayomnantes furent construitcs. Lne deuxiome campagne de tralaux clébuta vers 1155 11 6o. lille comprend l'exécution du déambulanoirce et des doux tours qui flanguent le choeur, chacunte accompagncée. (n) retour sur le manscpt, diune lravée d'escalier menant aux tribuncs du cheret ; l'étage cles tours fut vouté mais scul le mur cxtcirieur des mibunes de l'abside full élcré. C"est lors de cettc campagne quion adopta le transept aux bras arrondis et une élévation avec triforium sous le niveatu des mibunes du chour. I a rose fur construite au cours de la troisicme campagne dont Sermour a élabli le déroulement en se basmo sur des critères stylistiques. I es travaux commencèrent a la lois clans le bas-coté nord de la demière mavéc double de la nef el au rez-dechaussée de lannexe nord du transept qui (omp)rend le porche et som portail ce la salle du revestière. Il est fort probable que l'ólage inféricur du bras nord du transept qui relic ces deux parties ful comstruit on même tomps. Puis les travalux arancerent dans les tribunes du choem en les pattics hatues du thansept nord.

A notre avis la salle du trésor dur être exćcurée on même temps que les ribunes du chom puisquiclle se trouve sur le même niveau el qu clle commumique directement arec ces tribuncs: de plus, sa présence offrail un gage de stabilité pour la construction des partics hatutes du bras nord du transept. Les lralatux continuèrent ensuile dans les partics haules du cheur el de labside puis au bras sud du transept (') à

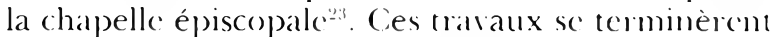
du côté sud de la demière mavée double de la nef.

Ainsi la rose qui éclaire la salle du trésor apparticut à la deuxicome phase des mavaux de cote moisième campagne de construction (de 1167 emviron à 1185 ). Seymour se refuse a préciscr la datation des dilférentes parties de cettc campagno à causc du caractère d'expérimentation du chantier et de l'extrême variété du décor ${ }^{2-1}$; cependant, pour notre propos il c'st esscnticl de préciscr la position relative de la rose dans le déroulement du chanticr afin d’en apprécier la forme el le décor el être ainsi en mesure de juger du caraciòe novatcur ou archä̈que de son modicle.

Pour l'analyse echnique de la rose du tréson nous utiliserons à litre comparatil les autres batics circulaires construites lors de la troisiome ampagne de construction. Comme la plupart des domnces lechniques impliquécs par sa construction se retroment durant tout le chantior. ces considéations semiront plus à préciser la structure de ce lrpe de rose et à definir le contexte anchitectural aupuel clle appartient qu'à préciser ici sal dallation. (On fera d’abord l'examen du cadre de ces fenêtres. en particulier de leur insertion dans la tratéc, puis de leur châssis de pierre, tant dans leurs élóments comstituants que dans leur apparcillage en leur ststème de fixation des verrières.

l.utilisation de fenêtres circulaires est cortaincment liunc des caraciéristigues de la troisiome campagne de construction bien (puc, dans l’élat actucl. leur importance soit beaucoup moins appanentc. ( )n renoure dans les parties construites lors de ce chantier trois rupes do fenêtres circulaires: l'oculus à la chapelle épiscopale, la rose polvibléc du bas-côté nord de la nef el la rose a colommentes de la salle du trésor. De plus un motif de jour circulare a découpe lobée fut également emploré pour omer les tympans des fenêtres de la claire-roic supéricure du transept et de la dernicie arave double de la nef. Le remplage des tribumes de la nef présconte aussi ce motil circulaire, le seul qui sera par la suite conserve dans les chanticrs ultérieurs de la ned. Il y a donc un gout prononcé pour le motil circulaire a llace lobé sm le chantier de la cathédrale de Noyon de 116 (i-7-70 à 1185 ce la rose de la salle du trésor apparât d’embléc comme appantenant à un enscmble décoralif colıéront.

la dermière travéc du bas-coté nord de la nef. où visiblement débuta la troisiome campangne, comprend une perite rose octalobé aujourd hui bouchéc (lig. j); celle-ci mesure $u$ peru moins de deux mètres de diamère () n a fail peu de cas de sa présence dans loordomnance du mur extérieur des bas-cotés de la nel car ceux-cionl subi d'importantes

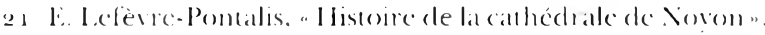

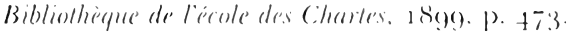

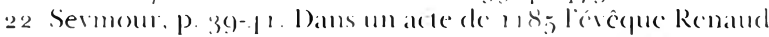
angmente le persommel de la callectrale el definie lems

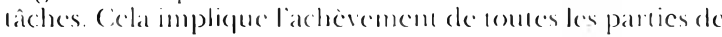

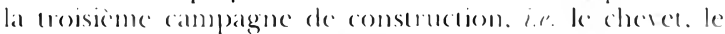

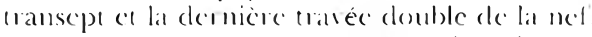

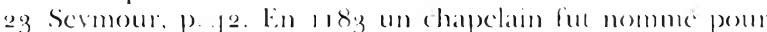

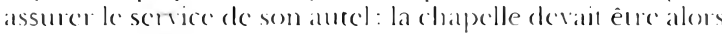
rerminice

24 Sermolli, p. p

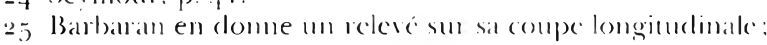

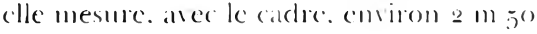


modifications à partir du XIV sicale ${ }^{2 t}$. Selon Seymour, l'ordonnance d'origine des bas-côtés de la nef présentait à chaque travé une suite d'arcatures aveugles surmontées d'une double fenceure" I a présence darcatures arcugles est attestée pour les deux dernières travées du bas-côté nord, mais elles ne devaient comporter sclon nous quine lenêtre simple, tout comme les travees du transept construites à la même époque. ('ost également le parti des travées de tribuncs correspondantes qui ne sont éclairces que par une scule lenêtre, à la différence des autres travées des tribunes de la nef ${ }^{2 *}$. La présence d'unc rose à la dernière lravéc du bas-côté nord aurait été motivée uniquement par lexistence d'une porte qui donnait accì̀s au cloître è bâtiments

26 Sermour, p. $5(1)-53$ Cl $8(1)$

27 Lne section d'arcature est conservée dans lanant-dernière marée du bas-coté nord de la nef construite vers a 1 go. L'ne porte a écé visiblememe percere par apres. Voir Comrauld Archives $3 / 11 / 50$.

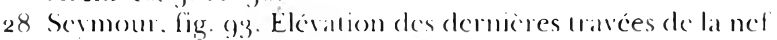
coté nord et comrauld Archires. 3 - 11 it

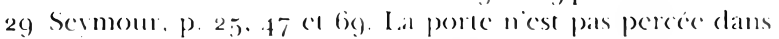
l'axe comtral de lat tavéce. comme la rose. mais décalćc vers la pile oucst. ()n reurouve la même disposition dans ies portes dutransept : pour les trois portes du mur oricontal du

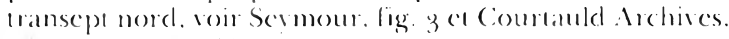
$311 / 79013: 1184$.

30 I) apres un releve de Barbaran, woir Sermour, figg. 21. canoniaux situés du côté nord de la cathédrale ${ }^{2 !}$. Cette dernière travée n'élant pas spique de l'ordonnance du mur du bas-côté, l'utilisation d'une telle fonêtre circulaire a clonc étć très limitée clans ke programme de la nel

I a position de cette rose dans le mur est cependant particulière: l'ourcrture de la baie ful conçue en relation avec l'arc formerer de la voûte qui la circonscrit parfaitement, et la largeur de la travéc entre les dcux piles constituc le diamètre horizontal du cadre de la rose dont la circonférence ne déborde que très légèrement sur le niveau des chapiteaux. On retrouvera ce type d'insertion dans la travéc à la rose de la salle du trésor:

Quant au profil mouluré de son cadre, unc étroite scction plate cntre deux baguettes, suivie d'un cavel dégagcant sur l'arcte un cordon toricjue, c'est une mouluration qui souligne la surface el non l'ébrasement de la baic. Ce traitement s'apparente à celui des oculi de la chapelle épiscopale et diffère du profil oblique beaucoup plus ćlaboré de la rose de la salle clu trésor.

Les oculi de l'ancicnne chapelle épiscopale (Fig. (6), reliée à l'origine au bras sud du transept et construite en même temps, présentont un deuxième type de fonêtre circulaire. Ils furcnt prérus sàns châssis de pierre et mesurent cnviron $\left.1, \check{5}^{(}\right)$m de diamètrc ${ }^{30}$. Ils

Figtre 5. Cathédrale de Noyon, rose octalobée de la dernière travée du bas-côté nord de la nef (Photo: Auteur).

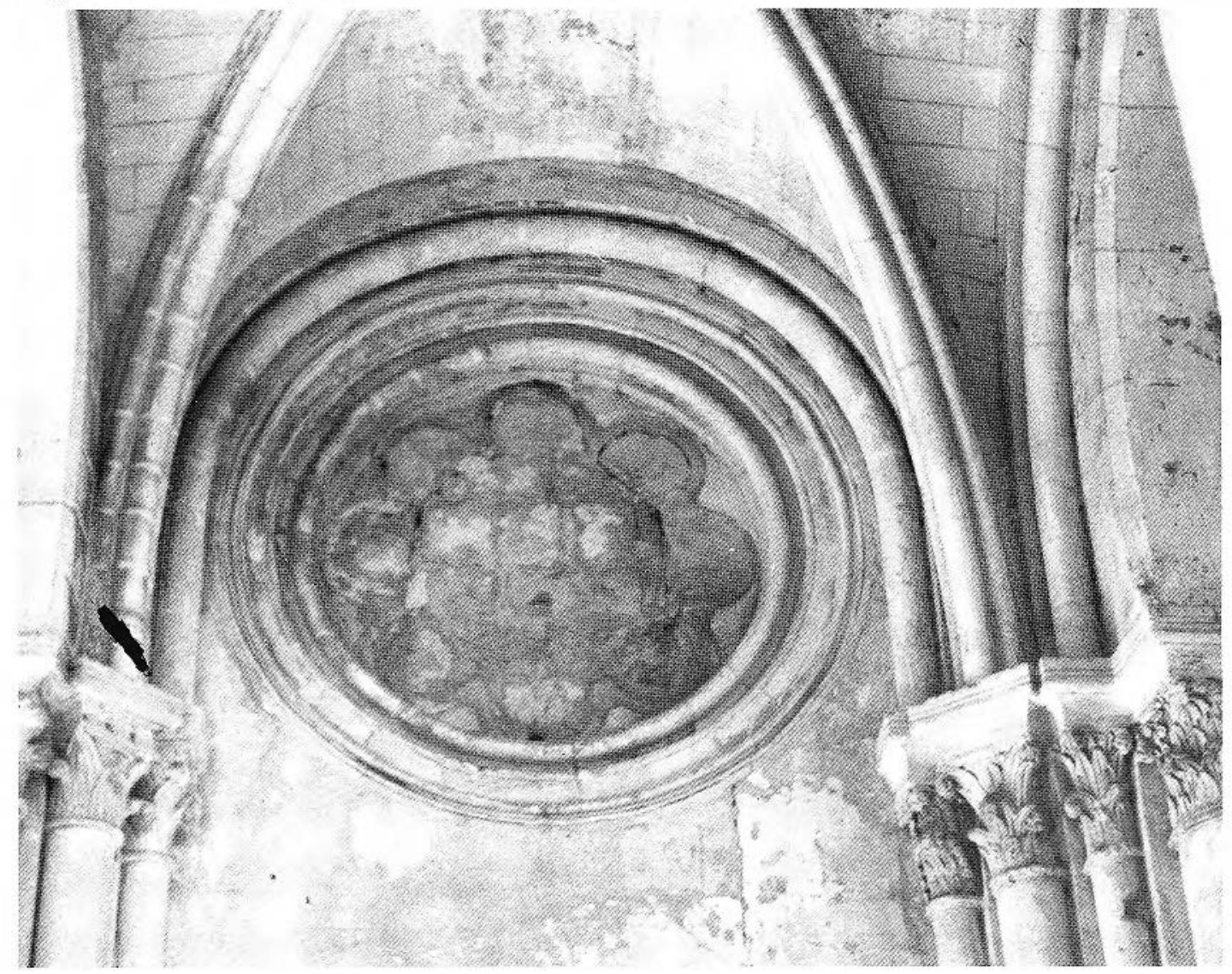




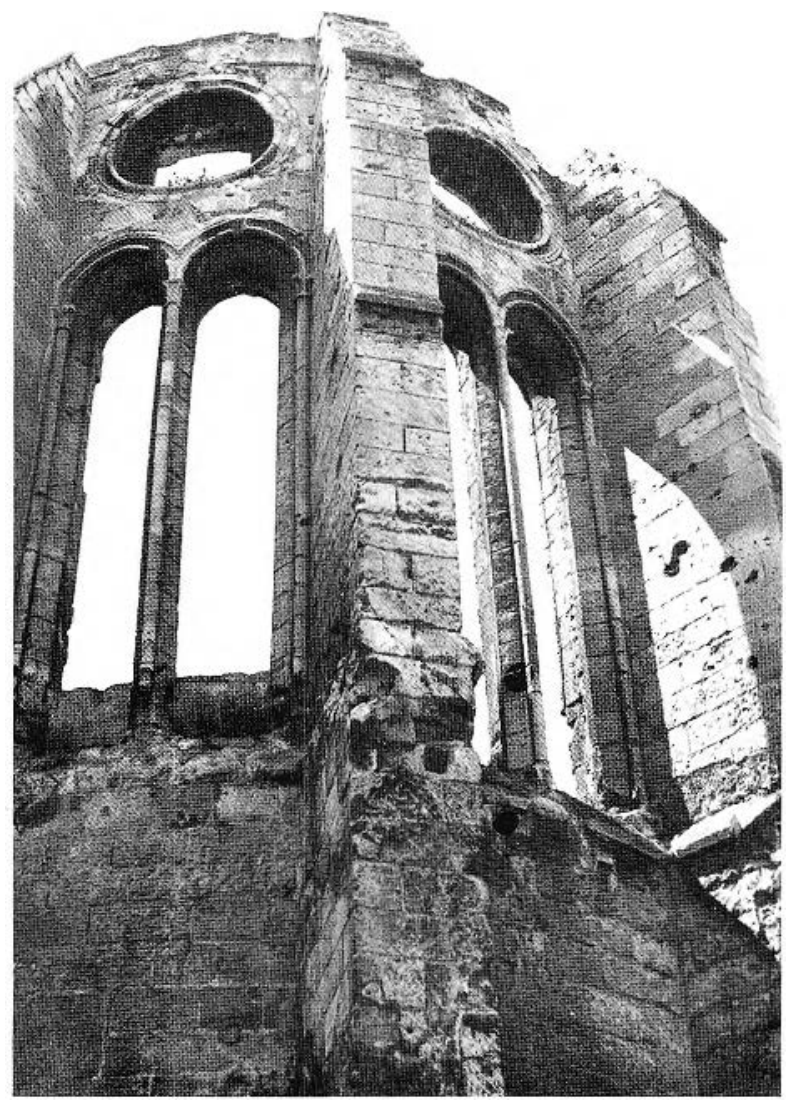

figtre 6. Chapelle ćpiscopale de Noron, oculi (Photo: Autcui). sont percés directement dans le mur el strmontent chacun deux lancencs. Bien quil soil difficile dianalyser leur lype d’insertion sous des volues aujourd'hui détruites, il semblerait, d'apros les traces observables sur des relerés et des photographies anciennes". (puc la position des oculi sous les voutes ait été différente de celle de la rose octalobéc. I.e cadre des oculi nélant pas immédiatement circonscrit par les voutanins. une portion de mur les isolani de l'are formerer des von̂les. Ces oculi auraient donc été d'abord concus en fonction de loordomance d'un mur qui, au-clessus d'une série d'arcatures areugles. comprenail à chaque travée deux lancelles surmontées d'unc fonôre circulare pour les regrouper.

I a rose a colonnelles de la salle du trésor constiuc le troisième rype de fenêtre circulaire de la cathédrale du xir siede (Fig. T). Iout comme la rose octatlobéc, clle ful aménagéce en fonclion de son insertion sous la voûte, ce qui entraina à l'extéricur unc position très decentréc dans loordomande du mur de façade. Fidairan la première des deux maves de cette perite salle. clle ajoure loutc la partic supéricure du mur entre les deux piles el sous l'are lomermet. C'est le même rapport proportionnel entre la rose el la travée quiau bas-côté nord de la nef. I ce adre de la baic comporte unc fenillure centrale entre deux cercles de clarcaux de même épaisseur el présentant le même profil. Cies cadtes forment un chrasconcon

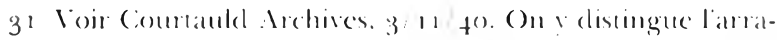
chement des atres formereds de l'hémicrele gui soml concen-

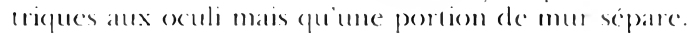

frourz: 7. Cathédrale de Noyon, la rose vue de l'intérieur de la salle du trésor (Photo: Autcur).

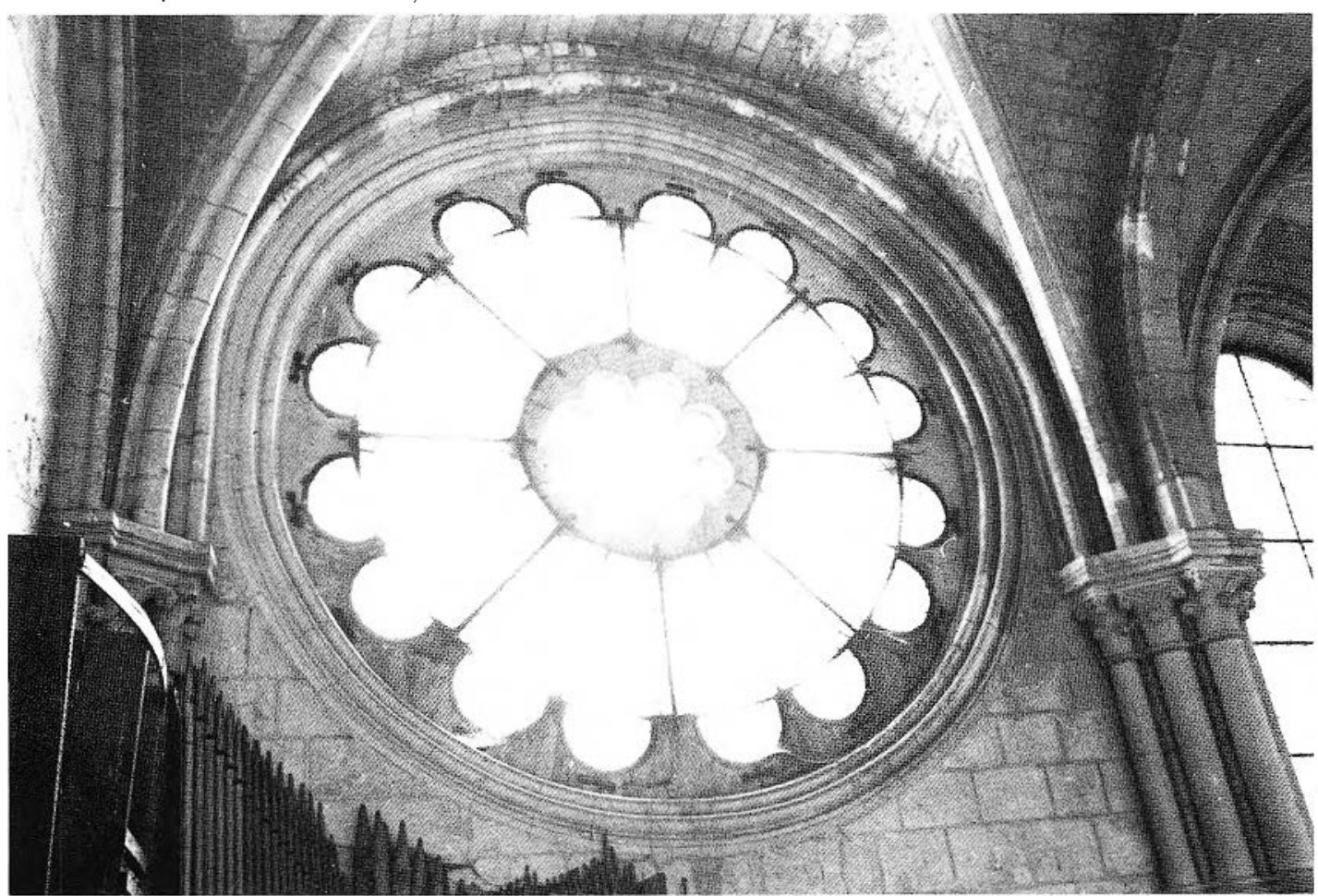


oblique dont le relief est accentué en (ompatraison de la modénature du transept: il s'apparcnte plus au tratement des partics hautes du cheur cl cle l'abside.

L'aménagement du vide circulairc ctant indissociable du gros de l'œuvre, nous sommes donc assurés de la présence de fenĉtres circulaires du débul à la lin du troisième chanticr. Cependant l'anénagement de l'ouverture n'impliquait pas nécessairement l'exécution immédiate du remplage de la fonêre. I e remplage de la rose du trésor a été inséré en second lieu; il faut maintenant se demander si les icohnicjues unilisécs pour l'exécution du châssis el le ype de décor déplové n’indiquent pas des licus plus spéciligues arec dautres parties du troisicme chanticr.

Le châssis de pierre de la rose comprond lrois zones concentriques de deux upes d'ócómoms: la dalle découpéc et la colommette con délit. I c promicr corcle est formé de 16 dalles ćpaisses. dícoupcés en claveaux redentés. Ce corcle sünscrit dircomement dans la feuillure taillée au tableau d'ourcroure de la baic-ce qui assure son mainticn dans le plan vertical. La deuxième zone comprend les 8 colomnences gui relient en les étrésillommant l'(eil au cercle périphóriquc. Lineil est également formó de 8 clarcaux reclentés.
L'emploi de dalles découpécs cu clarcaux redentés fut constant au cours du troisiène chanticr. ()n les retrouve à la petite rose du bas-coté nord de la nelou elles forment un châssis octalobé semblable a celui de l'ceil de la rose du trésor. I ce claveau redenté fuu également employé pour domncr unc forme rrilobce aux jours circulaires qui allègent el ornent lés tympans des fenêtres de la claire-voic supcicionc du transept (Fig. 8) et des demiones travées de la nef. Il apparaît aussi au remplage destribunes de la nefle Ces jours trilobés n'ont qu une fonction onnementale. I a frise d'arcades trilobées du choum est forméc d'un autre sype de dalles découpées (Fig. (9). (ies dalles rectangulaires auxquelles on a dégagé, a la partic inféricure, une forme tribloée. somt juxtaposćes en frise, et sous leurs jomts verticatux appanaissent de courtes colonnettes qui accentucnt le dessin des arcatures. Leur mode diassemblage est tres simple (at aucune pression ne sexerce sur ce décol plaqué ou les dalles sont rattachées au mur par unc anconde

Les colonnettes de la rose somt utilisées selon $\mathbf{~ m}$ principe différent. Elles rencontremt les dalles extérieures du remplage sur la pleinc ćpaissenr du redent. En effet, si la colommence en délit est un ćlóment 3. Setmour. p. 73.

Figure 8. Cathédrale de Noyon, trilobe dédoublé du tympan des fenêtres de la claire-voie du transept sud (Photo: Autcur).

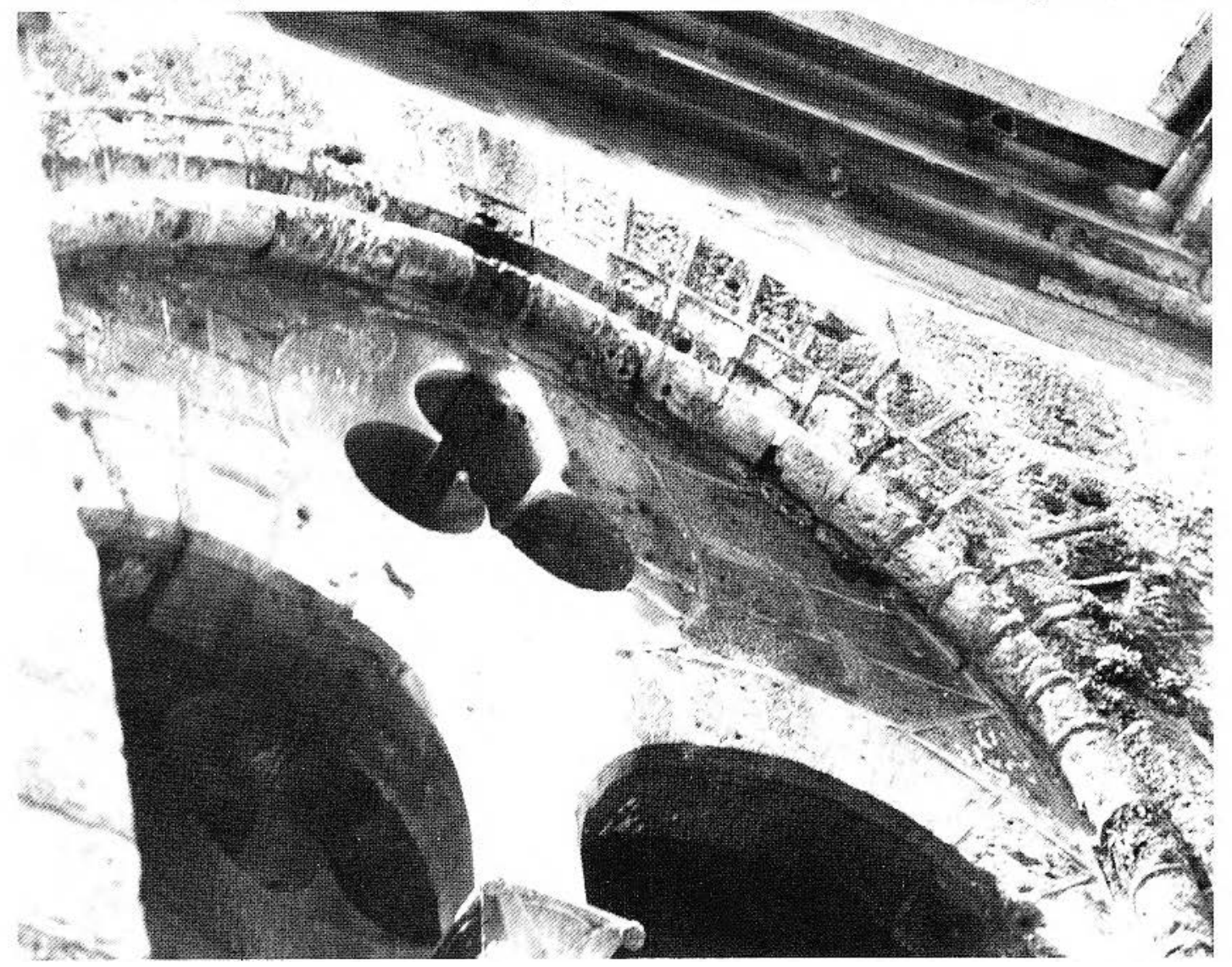




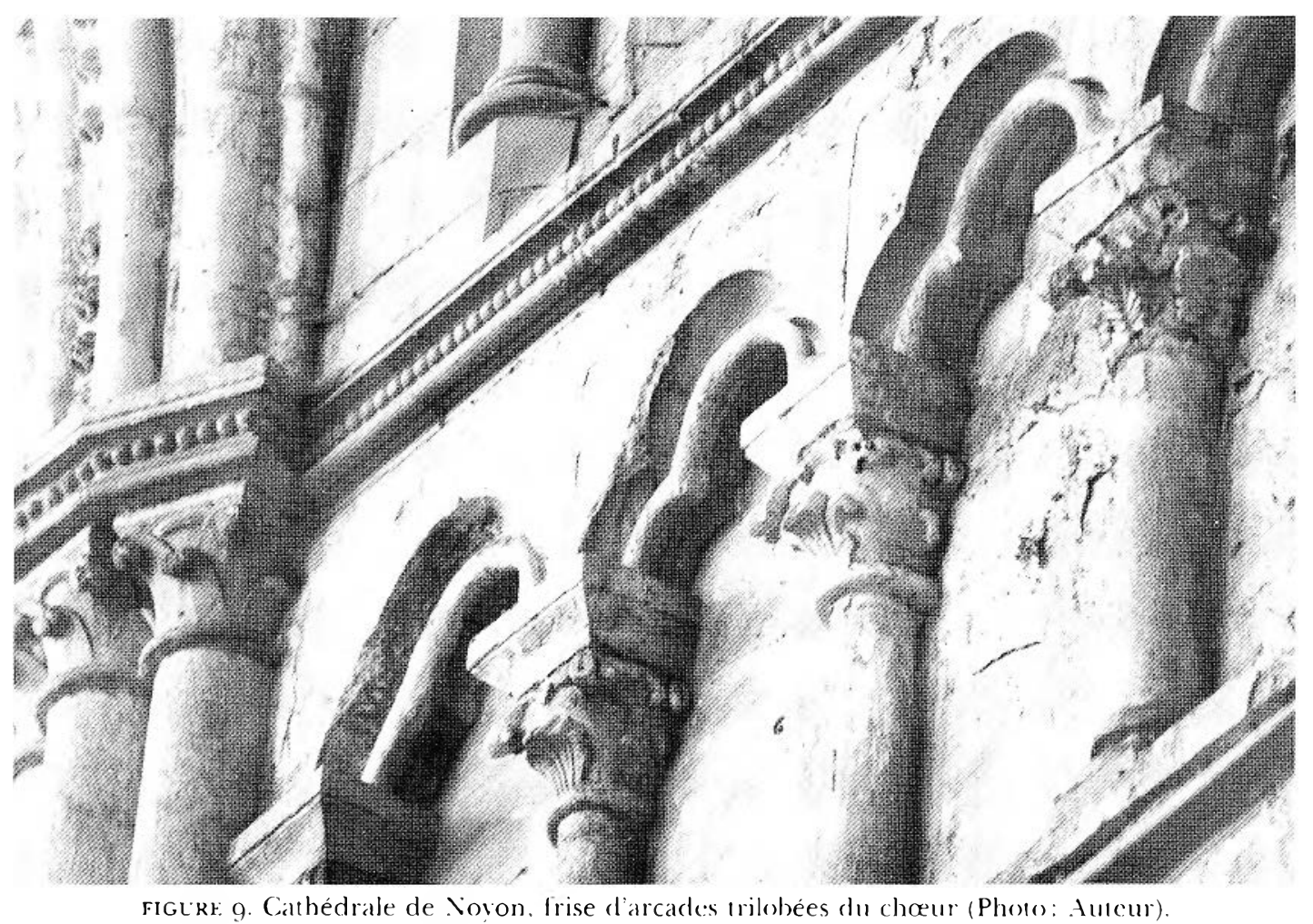

caractéristique du décor de la calhédrale de Noyon, on ne retrouve qu’à la rose des fûts con dólit employés comme étrésillons". In reliant les deux corcles de claveaux, les colomncules assurent le raidissement du réscau. ce qui ess le gage de sa solidité.

l.apparcillage des ćléments du châssis a ćté soignousement ćlabli: les bases des colomncues prennent appui sur la ploine épaisseur de l'cxuados de l'ocil et leurs tailloirs rencontrent les dalles périphériques en leur centre, sur un redent ćlargi pour assurer un solide joint perpendiculaire. 1)e même, tous les joints des claveaux de l'oeil e du pourtour sont posés sur des rayons différents du cercle. Ce lracé régulateur simple et cllicace et les proportions trapues des ćléments domncont à la rose un aspect un peu archaíque. En clfet, le réscaul de la rose comtraste avec les proportions élancéés el la minceur relanire des élémonts des fencolres supéricures du transept. I)ans le: transept. on a disposć a chatpue travée. dans $111 \mathrm{~mm}$ dédoublé, une clouble fenêtre inscrite sous un are de décharge dont le trmpan ajourć d’un urilobe prélïgure la fencetre composée du gothique dassique. Mais ici aussi la technique de comstruction du remplage restc traditionnclle. atr malgré la finc colonnetuc allongée qui rémit le cinne des deux lancentes, le tympan peu ourert reste formé d'assises horizomales el de claveaux pour les cintres. L'n seul mencall est utilisé pour farre la liaison contre les deux cintres des lancettes: c'est une pierre de forme pentagonale (Fig. 8)
Les verrieres qui ornaicnt à l'origine la rose ont disparu depuis longtemps mais la lencure ('n a conservé le mode de fixation. I. remplage de la rose avait pour lonction de supporter les panncaux de rerrières. (On commatit très mal le mode de lixallion des vitraux des premières roses car les plus anciennes ont généralement subi des réannénagements postéricurs. Ainsi, la rose de Samt-Fiennc de Beaurais porte maintenamt sa vitrerie dans l'épatsseur de son réscau où des rainures onl été creusées pour recoroir les vitraux (Fig. 10). (ies raimures imitent les lenillures cont on allait munir les châssis de picrre a partir de 1220 comiron. A la rose de Noyon, la présconce diun décor sculpó sur l’épaissour du réscaut a sans doutc ćpargnci un lol réaménagemont el on pent encore aujourd'hui obscrver l'armalure scellée a la lace intéricure du châssis. latissant les colomncttes du réscau libres.

L'armature actuclle scmble d'origine (Fig. 7). Fille comprend of ballotières rayommantes sappuyant d’uncôté à l'(eil, où un cerrele de métal les réunit, cr de lautre côté sur les 16 redents périphériques. où un second cercle les mainticnt en clélimitant d'une part if demi-cercles a diautre parl 16 linseaux ravonnants, formes probables des pammeaux d'originc des

33 Scymomr. p. 101-1012

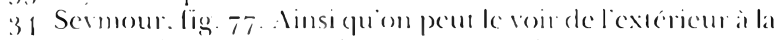

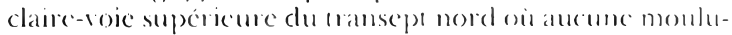

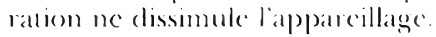




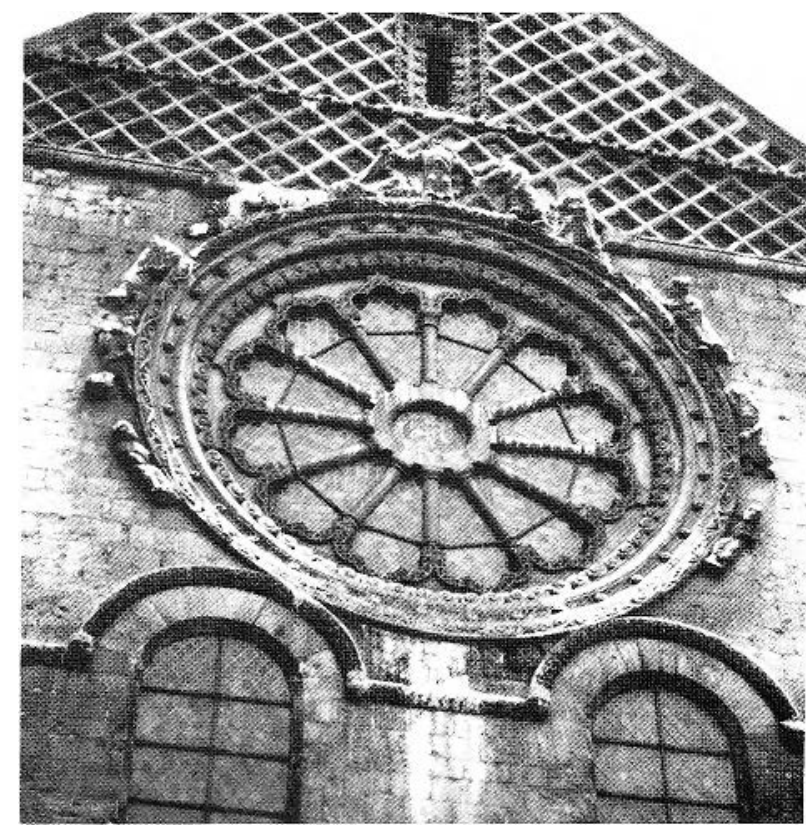

FIGLRE 10. Figlise Saint-Fienne de Beaurais, roseroue de fortune. vers 11 jo (Photo: Aulcur).

vitraux. I'xeil octalobé portc dans l'ćpaisseur de ses dalles un petio cercle (pui délimite à son tour 8 petits demi-cercles autour du médaillon central. I.es verrières étaient ainsi posćes sur deux plans différents: inscrites dans l’éparisscur de l’oxil ou accolées à l’arrière du remplage, laissant les colomnetles du réscau libres. Cotte techmique de lixation des verrières sur deux plans dillérents est celle qui scra praticjuéc au début do la lenĉre gothique composéc. I a rose de Novon témoigne quine telle pratique existait auparavant. Le rapport de l'armature au remplage est également révélatcur: le pourtour bilobé de la rose napparaît plus commo un simple ornement, le rcdent libre offrait un appui supplémentaire pour lixer les panncaux de verrières.

I 'analyse technique de la rose, son percement dans le mur él'exćcution de son remplage ne permettent pas d’élablir des liens plus étroits arec d'autres parties de la cathédrale du XI' siècle. Lce troisionne chantier utilisa la fenêtre circulaire, la dalle découpée en claveau redenté el la colomnette en délit du début à la fin de la construction. C'est l’étude du décor sculpté qui permettra sumtout de préciser la datation de la rose du trésor. L'examen de sa mouluration. du décor de feuillage er de fleurs des chapitcaux et des frises, ainsi que les masques de l'archivolte pornertenı de situcr la rose all débul du troisiòme chanticr. vers $1170-1175$.

35 Sermour. fig 121

36 Sermour, tig 72 ia

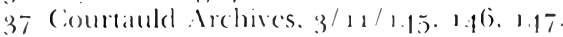

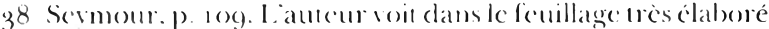

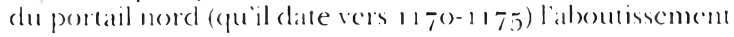
du decor du a atclier.

39 Courtauld Archives. 3/11/65. pile 31 (1 3/11/64 pile 2(9).

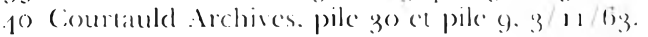

I a rose du trésor a reçu un décor très élaborć ct sa configuration est entièrement redessinée par unc mouluration soignée. Une ornementation régétale complète le décor de cette solide roue d'arcades bilobées (Fig. 2). La rose est mise cn valeur par une modćnature basée sur l'cmploi de cordons toriques bien dćgagés par des cavets. I.es profils sont posés de manière à accroîtrc la plasticité de la fenĉtre: les doubles cordons toriques des cadres intćrieur et cxtéricur sont dégagés sur un angle oblique et forment l'ćbrascment de la baie. Un tore central entre deux plus petits modulent la surface de l'ceil, faisant apparaître le moyeu de la roue. Cettc mouluration est fortement misc en reliel par l'amincisscment des redents de l'oul. Cie profil s'apparente à celui des bagues qui scandent la montéc des piles accolées aux murs intéricurs du chøur et du transept ${ }^{35}$. Le pourtour festonné de la rose cst également souligné d'une double mouluration torique qui profile l'arête des dalles périphériques à la lois en surface, où l’écoinçon évidé de chaque redent préscnte cn réserve une flcur, ct à l'intrados, où le cordon torique délimite un décor sculpté sur l’ćpaisscur des lobes. Cettc grande plasticité contraste fortement avec le décor du transept mais se rapproche du traitement des parties hautes du chøeur où l'on retrouve un même type de mouluration et les mêmes techniques d'exécution du décor: la réserve de motifs sur fond évidé, au bandeau supćrieur ct aux doublcaux du chœur, et un décor disposé sur l'épaisseur des dalles comme aux arcades trilobécs du chevet (Fig. 9).

L'ornementation de feullage de la rose, de grande qualitc. permet des rapprochements plus précis avec d'autres parties du troisième chanticr. Différents motifs de feuillage apparaissent aux chapitcaux, aux redents et à l'archivolte de la ficnêtre. I.cs chapitcaux actuels de la rose reproduisent ceux exécutés au XIX siècle, sans doutc d'après les modèles d'originc. En effet, bien que nous nayons aucune garantie documentaire de leur conformité, leur style correspond parfaitement aux autres clonnces relatives à la rosc et confirme sa position dans le déroulement du troisicme chantier. I.es chapiteaux de la rose sont d'un modèlc apparenté à ccux de la sallc du trésor, modèle qui réapparaît, un peu plus développé, dans les partics hatutes du transcpt nord ${ }^{3 i}$. Les chapiteaux préscutent un type de feuillage d'abord utilisé à la fin de la deuxième campagne clans les murs cxtéricurs des uribunes du chocur ${ }^{3 i}$, avant d'ôre développé avec une très grande richesse au portail nord (Fïg. 11$)^{3 x}$.

On retroure les mêmes feuilles sur les chapiteaux des dernières travées du bas-côté nord de la nef où, à côté de ceux lortcment inspirés par le décor du portail ${ }^{3}$. apparât avec cc même type de feuillage une premicre version plus architecturale (Fig. j)" La bordure de la corbcille y cst claircment délinie sous l'abaque el le tailloir, el de longucs leuilles lobées s'cnroulent en volutes feuillucs, inscrivant de courtes feuilles lobées debout sur leur tige. C'est ce type de chapitcau qui fut utilisé à la salle du trésor où, malgré 
les restaurations de Selmersheim, nous sommes assurés du modcle d'au moins un bloc de chapiteaux". Sur ces chapiteaux aux proportions plus graciles, les mêmes feuilles lobées s'inscrivent sous dos fouilles d'angle qui sc recourbent sur des fruits d'armm ; dans l'axe central deux autres tiges souples s inclinent arec leurs fruits d'arum. ('e motif appartient au décor du portail mais n'apparâit pas aux chapiteaux actucls de la rose. I.c traitcment de ceux-ci est un peu simplifié en raison sans doute de leur échelle: on y renoure les mêmes feuilles lobécs qui projettent cottc fois des corolles de lleurs". (Ce motif est présent all portail sous la rose mais fut utilisé auparavant a la fin de la deuxième campagne; il réapparaît dans unc autre série de chapitcaux, d'un type différemt, au mur intéricur des tribunes du chœurr.13.

Le décor des chapicaux confirme la marche des travaux du uroisiome chantier telle quélablie par Seymour: d'abord le bas-coté nord de la nef ce le rez-de-chaussic de l'annexe du transepl nord arec son portail. puis les tribumes du chour, la salle du trésor el le bras nord du transcpt.
Un critere stylistique, complémentaire à l'ćtude des chapiteaux, permet de regrouper diffóremment les parties du troisiome chantier et précise la position de la rose: c'est celui de l'articulation des piles composées qui recoivent les roûtes. I ce parti adopté lors des promiores campagnes de construction plaçat les supports recevant les ogives du volutement on diagonale sur la pile composée. logive tombant perpendiculairement sur ces tailloirs obliques : la centralité de l'espace de la travéc se trouvait accentuce par le voutcment". (ie parti ne sera abandonnć qu'au

41 Barbaran, delails de perspectire: chapilcalux du lleson angle sud-est. Dessin exécute avant les restantalions de Selmershoin commencécs en 1873.

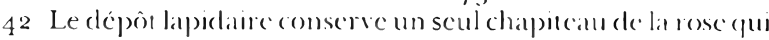
apparticul a ce 11 pe.

43 Courtaukl Archives $3 / 11 / 1$. 0 et $3 / 11105$

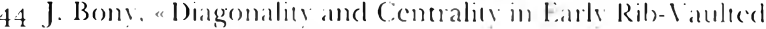

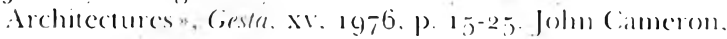
Oakland L niversits. a cexposé lors de la quatrieme (antatdian Conference of Medieval Art Historians. I niversiry of

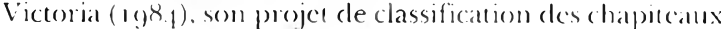
de la cathedrale de. Voson on il utilise ce critere.

figCre 11 . Cathédrale de Noỵon, annexe du transept nord, portail Saint-Pierre. délail cle l'ćbrasement droit (Phoso: Auteur).

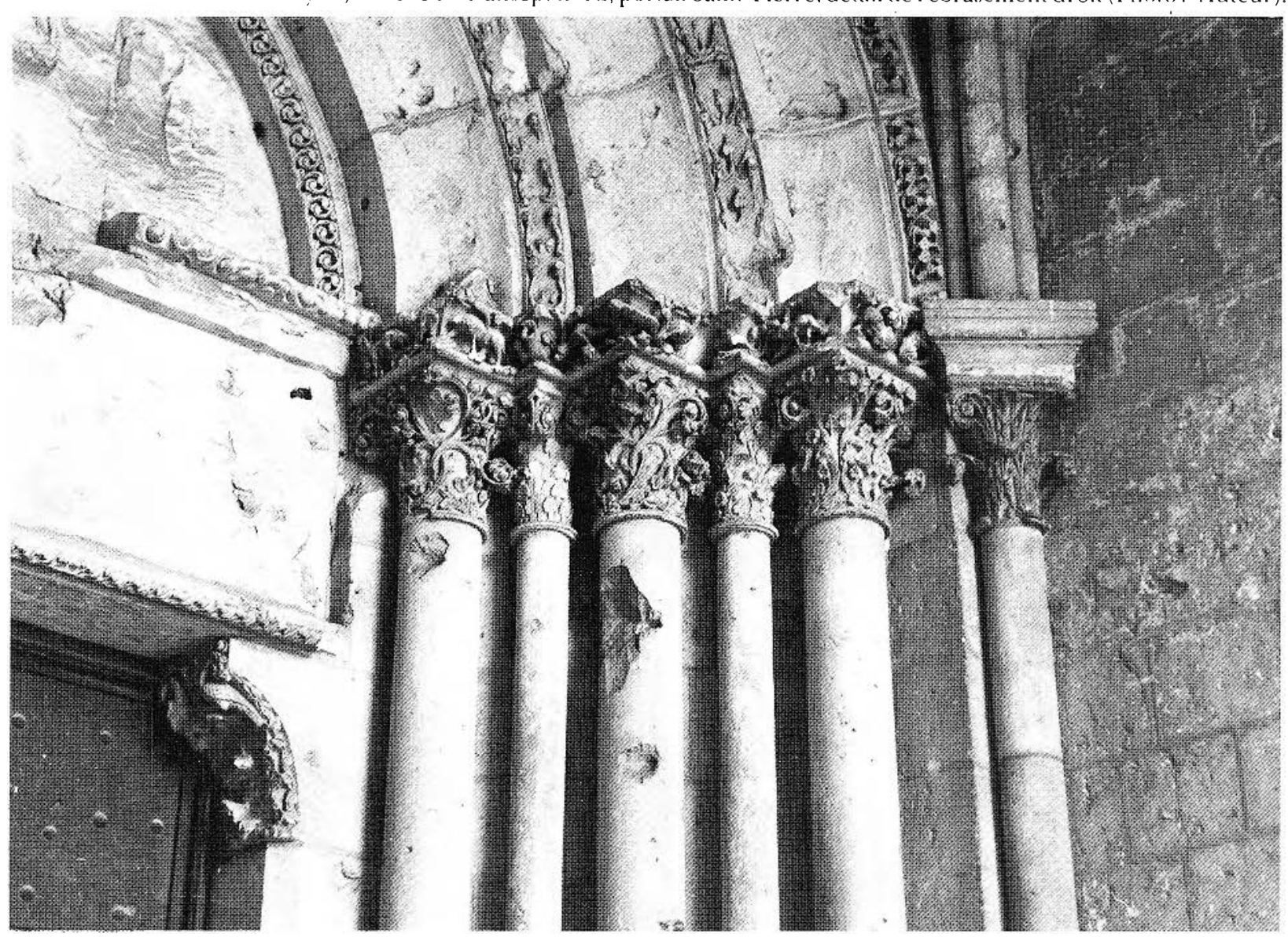




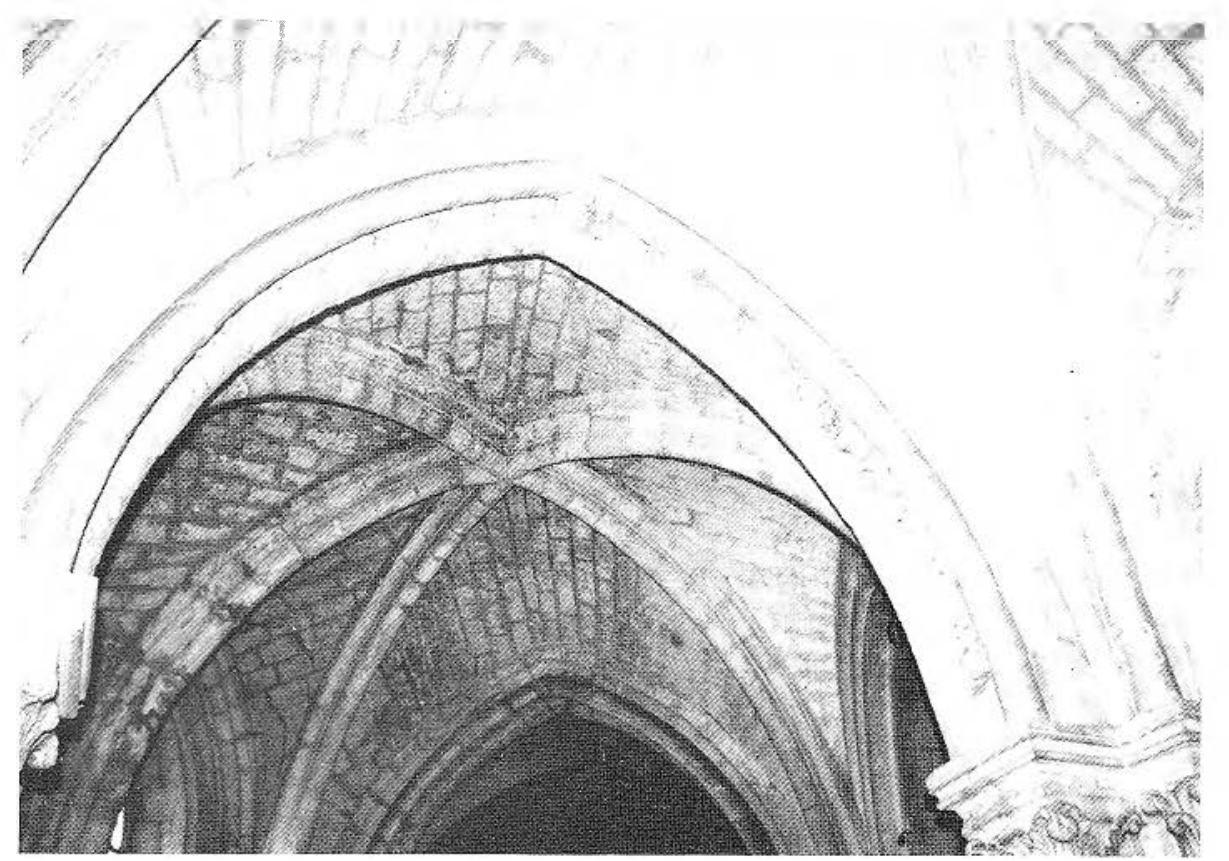

FIGURF. 12. Cathédrale de Coyon, doubleaux ornés de masques, tribune de l'abside (Photo: Auteur). cours de la troisiome (ampagne de comstructions : on unilisera alors une pile composé de supports disposés à angle droit ce formant des ressauls réguliers. ceux-ci recerant diagomalement les ogives (Fig. 12).

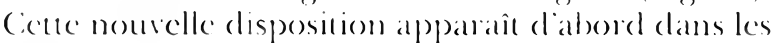
piles intéricures des mibunes du chemen où elle

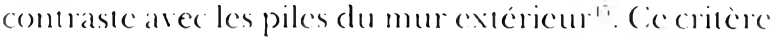
place la comstruction de la salle du trésore domtes

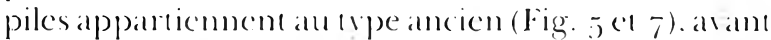
lacherement des rribunes du cherur. (ies deux parties présentent on cffer $m$ momratu profil d'ogive a

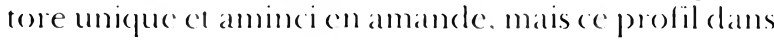
la salle durés() apparticmt à un ensemble strlistique boaucoup plus cohóremt. La salle du trésor précécla

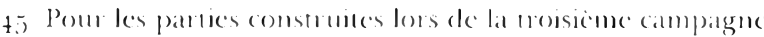

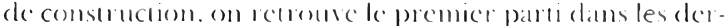

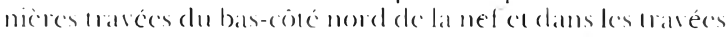

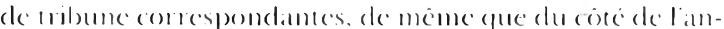

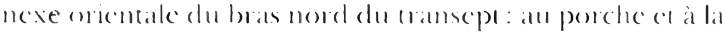

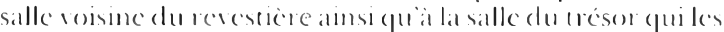

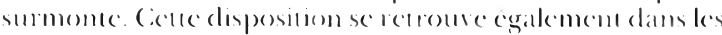

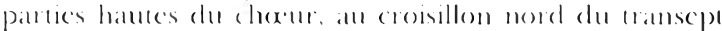

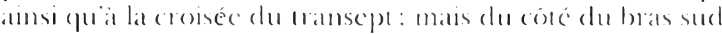

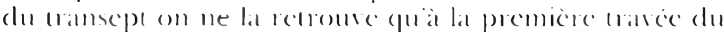

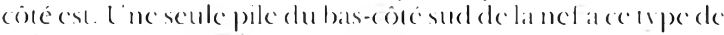

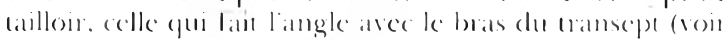

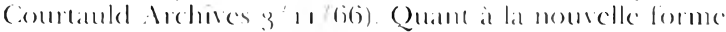

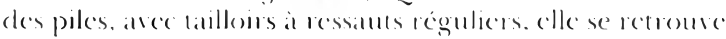

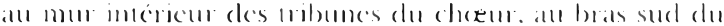

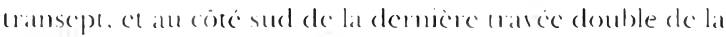

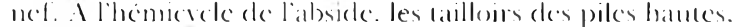

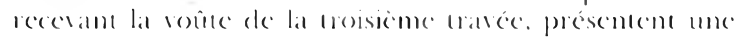

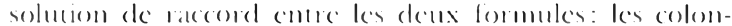

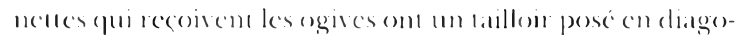

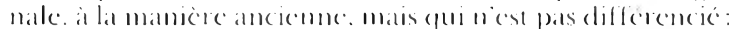

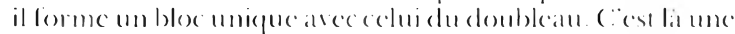

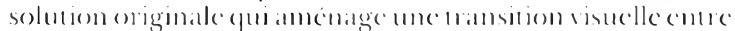

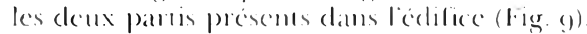

raisemblablement lachovement des ribunes du cheur. et l'exécution de la rose. colle des partics hatules du chemen ou lion remoure un clécor apparrenté.

Les redents de la rose sont ornés de corolles de

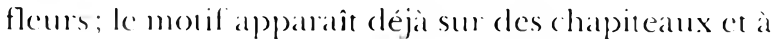
la console de la pile nord-oucs de la croiséc du transept. mais le tratement des fleurs de la rose les apparente plutôt a celles qui décorent les ogives de l'ab)side. Lonncmentation de la rose comprend finalement deux rinceaux de feuillage (Fig. 2). Des suites deperites fouilles lobées, alignées sur leurs lige's, sont sculprécs sur léparissem des redents. () n retrource même décor a la bordure inféricure du linteatu du portail nord (lïg. 1 1). I allohivolte de larose présente un sccond rinceatu beatucoup plus riche: son profil tres arrondi fail sallie sur le plan dumur et accentuc l'ébrasement de la baie. Il est comstituć a chapue clarcau diunc baguche centrale ornéce en alternance de perites fenilles dentés ou d'um galon de trous cubigues; de parte et dautre sopposent urois bouquets de fenillage, les bomquets cemmanx inscridam des mascarons. (e rincean constitue un motif original qui sapponente au décol du portail nomel. ()n renource en efler six motils différents de rinceatux: deux bordemu le lintean ce quatre séparent les voussures de l'archirolte, aujourl'hui privées de leur dé(o) historice. Le rinceatu de lanchioste de la rose présente un même répertoire de feruillage son $1 \mathrm{ppe}$ de (ompositions se rapproche du rincealu extéricur de larchivelte de portail (Fig. 11).

Ln dernier ćlément marque concore lappartenance de la rose an moisiome chantier. Le décon secondarie de la calléelrale de Joyon est célèbre par son emploi de têtes humanes associées a des ćlé-

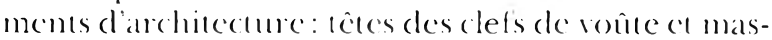


carons des arcs doublcaux des tribunes du chour (Fig. 12), têtes et mascarons des consoles des piles ouest de la croisée du transcpt, têtes en corbeau des tailloirs des piles de la nef et modillons de la corniche du chevet". Ia présence de petites lêtes humaines souriantes ou grimaçanı à l'archivolı de la rose est pratiquement inconnue. I.échelle de ces têtes implique ici un caractère plus orncmental que symboliquc mais leur existence constitue néanmoins un élément décoratif très intéressant qui, probablement peint à l'origine, devait être bcaucoup plus visible. Il est cliflicile de préciscr le style de ces têtes car le segment inférieur de l'archivolte, qui est d'origine, est très dégradé, ct si l'on peut ça ct là deviner lcur préscnce, on ne peut les analyser. I.c dépôt lapidaire conscric cependant un fragment de claveau oil liune de ces têtes est bicn conservée (Fïg. 13). On y voit un visage arrondi avec des yeux aux coins extéricurs rabaissés, un peu à la manière de cortaines têtes des tribunes du chœur. Mais l'exécution de ces têtcs est différente; elles montront une technique rapide csscntiellement ornementalc: seules des facctes oblipucs forment les arcades sourcillières, dégagent les joucs; la facture y est sommairc.

Ainsi, la rose de Novon révèle unc forme importante du décor de la cathódrale. Elle šimpose au scin du troisième chantier comme un ćlćment intermćdiaire entre l'exécution du portail nord et celle des parties hautes du chour. Son riche décor de feuillage et le relief très accentuć de son châssis lrapu prodıiscont une forme qui paraît découpée par le décor. ('e traitement inaugure unc tendance qui mène à un certain type de décor de la phase classique du style gothique (Plate Tracery) quillustre bicn la rose onest de Chartres (Fig. 14)

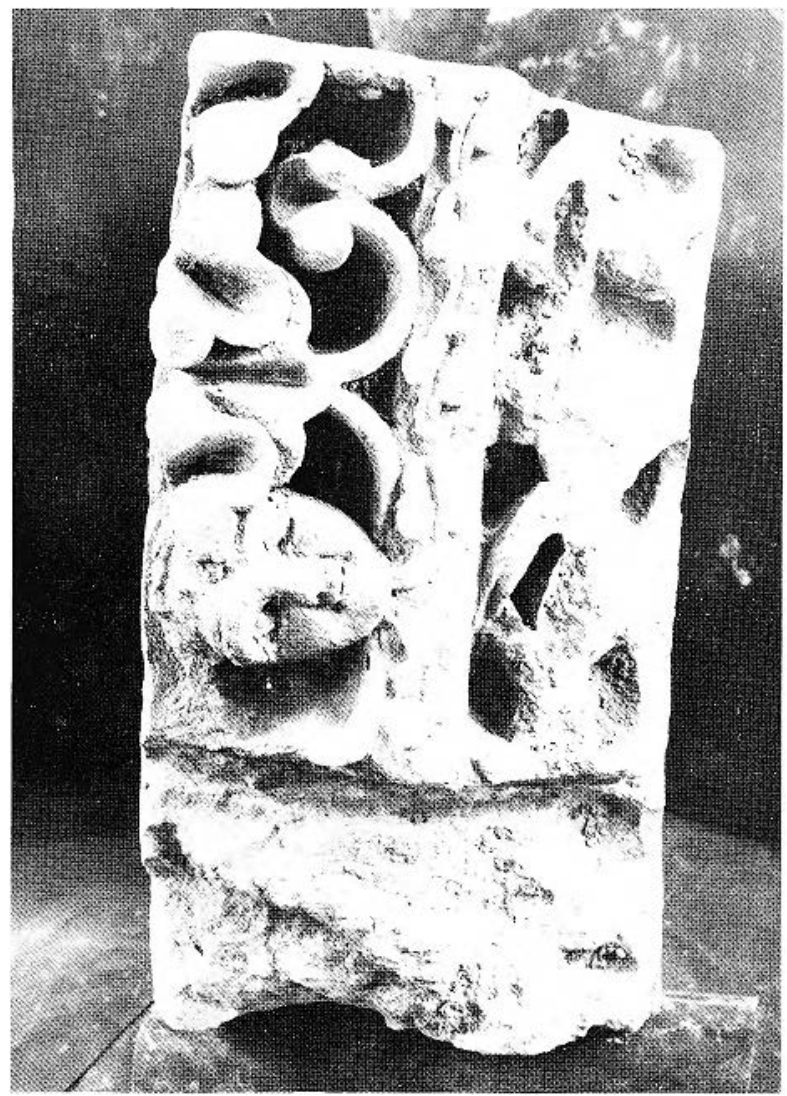

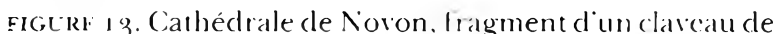
l'archisolte de la rose. dépôt lapidaire (Photo: Mrch. Mon. Hist.)

$4^{6}$ courlatuld Archives, 3/11/67.3/11/68. 3/11/71, 3/11/60 a $3 / 11 / 67$. Sermonir. lig. 15. Vorr aussi ( "Têes gothiques de lat cathédrake de Voron". Conselle des Bermux-1rts. 1937, p) 137-1 $t^{2}$.
Houre 14. Cialhédrale do Chartres, ditail de la rose ouest. vers 1210 (Photo: : Iuteur).

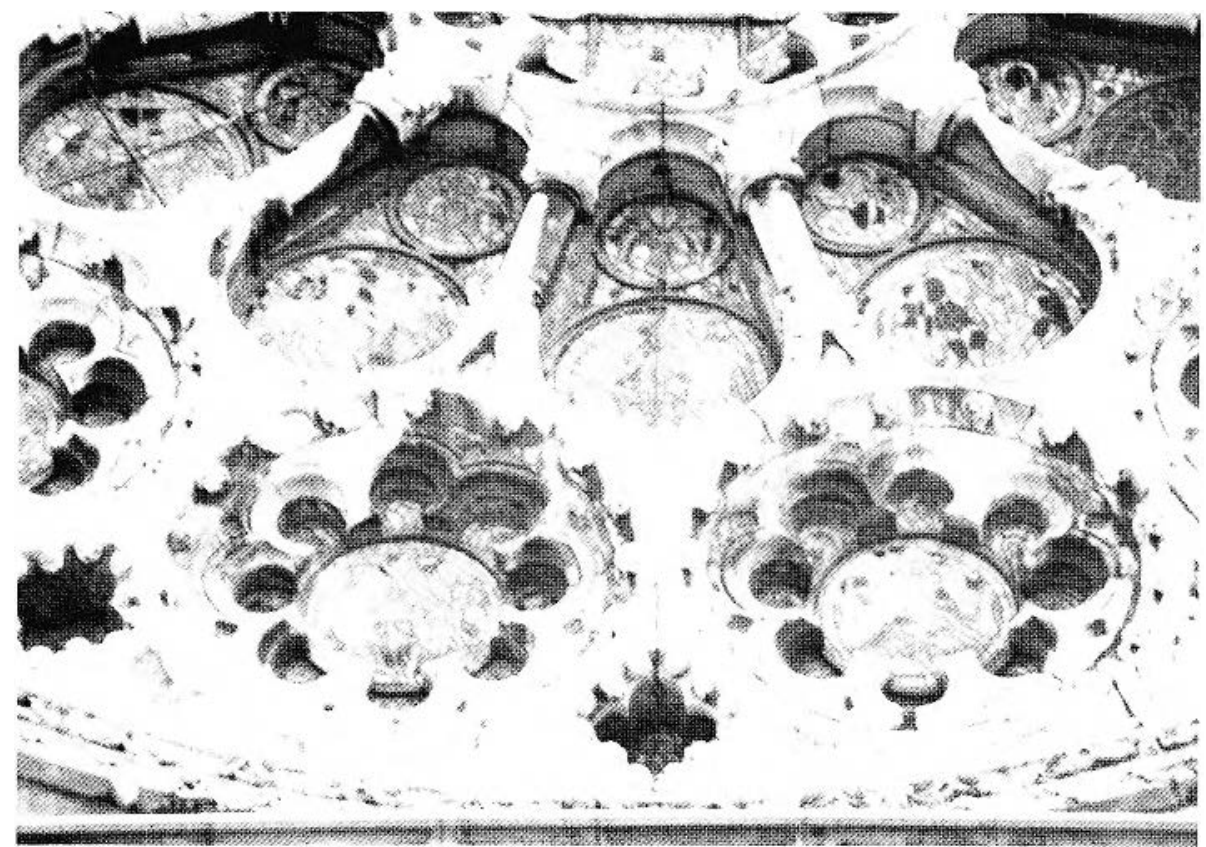




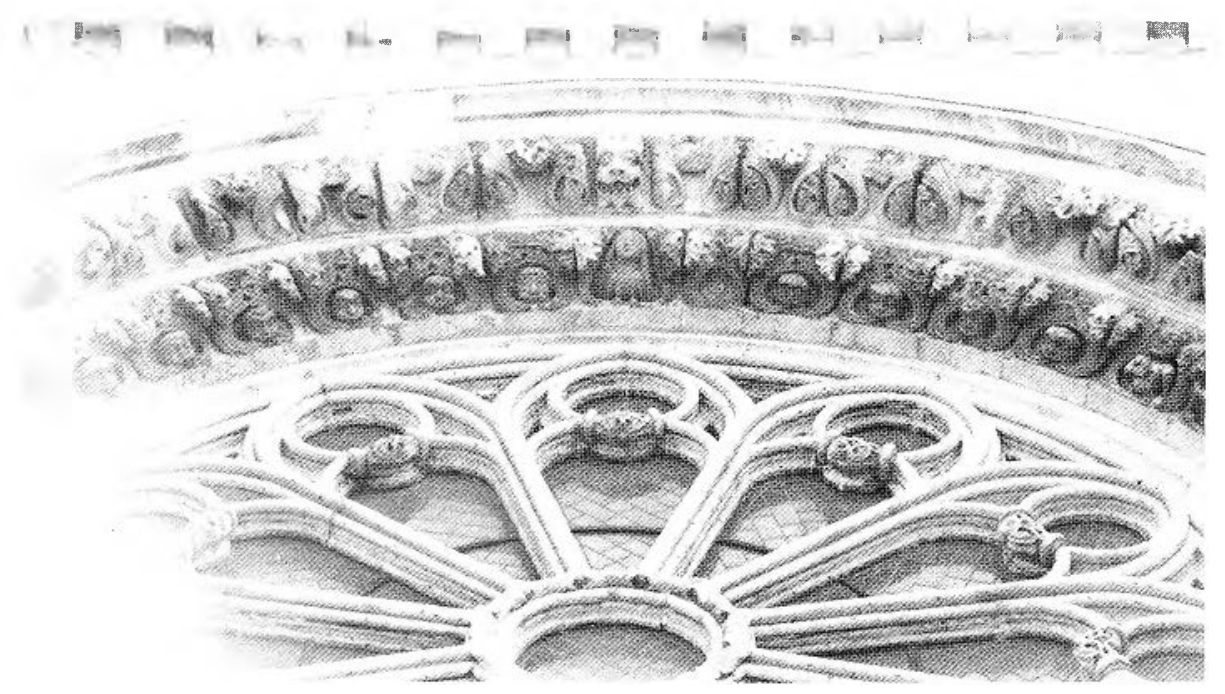

FIGURE 15. Abbatiale de Saint-Denis, détail de l'archivolte de la rose ouest. vers 1140 (Photo: Auteur).

I a présence de mascarons à l'archivolte de la rose s'explique par son appartenance au décor secondaire de la cathédrale: mais ils l'inscrivent également dans la lignée du décor des roses plus anciennes de SaintDenis ( 1140$)$ (Fig. 15) et de Nomc-Dame-en-Vaux de Châlons-sur-Marnc ( $1150-60)$, où le theme a cependant reçu unc importance plus grande. I e traitcment plus décoratif du motif des masques à Noyon traduit l'colution que subira ce type d'ornomentation au cours des décennics suivantes. Cette tendance était déjà perceptible lorsque les masques ćtaicnt utilisés à l'archivolte des portails' el saccentuera lorsque ce décor se répandra aux archivoltes des fonêtres ct aux cormiches des églises secondaires de l'île-de-France ${ }^{\text {tr. }}$.

$$
* * *
$$

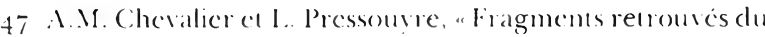

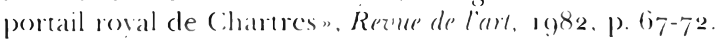

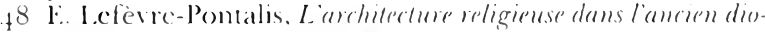

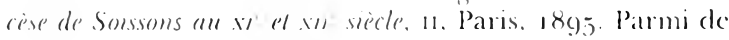
nombreux exemples notoms les maspues de l'archioble des fonêtres de l'abside de l'église de Berzy-le-Sec (p)l Xxir. 2), de la corniche de léglise de Commelles (p) 1.x. 2-3-1) et de colle de l'eglise de Montign-Lengran (pl. 1.xxv 1-4-5).

49 I.c motif lobé appanâ̂t tant dans le's plans que dans l'élévation. Le cheret ed le lransept forment un plan trilolex: cest cyalement selon un plan trilobe que sont disposices les colemenctes qui subdivisent les ouvertures des mibunes du (hoxur. Dans l'élévation des rrilobes ajourcontes remplages

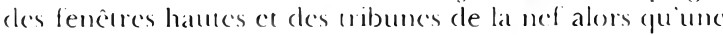
arcature arreugle trilobée orne le pourtour du sanctuaire

5) ()n fait remonter l'ensemble de la construction de Mons-

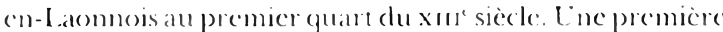
(ampagne comprend le cheres, (lont le plan dérive de l'al)batiale de Samt-Yied de Braine consalcrece en 1216 . I.

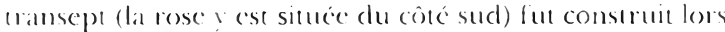
diunc deuxicme (ampatgne: il a cles voutes sexpatrites (omme atl choeur, mais les détails de som orncmentation somt plus recents (I.. Broche, "I. église de Mons". Congres archéologique de France, $1811,1 . \mathrm{p}$. jog-400). la modénature de la rose indigue lat fin du premier quart du xur sicele.
La rose de Voyon appartient au type le plus ancien de rose à colonnctres libres qui allait disparâtre vers 1180 . Mais cette lenĉtre présente déjà des modilications significatives par rapport à la rose de SaintFticnne de Beauvais (1150). Il n'est pas surprenant que sur un chantier oil fut cmployé à la fois la rose à dalle et la rose à colonnctte, celle-ci ait été influencée par la forme lobéc, forme privilégiéc par le maître de Novon". Celui-ci introduisit pour la première fois une forme octalobéc à l'oeil de la rose, auparavant circulaire, ce qui permettait d'agrandir l'(xil tout en lui donnant une forme plus décoralive et en assurant un meilleur soution des verric̀res. 1)c même, dessinat-il un pourtour bilobé qui augmenta l'effét décoratif tout en offrant des surfaces d'appui supplémentaires à l'armature supportant les verrières. Ces disposi(ions scront retenues par la suite dans l’élaboration d'un autre modìle perfectionné qui apparaît vers $1180-1200$ et dont la rose de Mons-en-Laonnois (lïg. 4) n'est qu'un exemple rclativement tardif "sa rose de Noyon nous renseignc également sur le mode de fixation des verrières de ces roses-roues et confirme l'importance de ce facteur dans l'élaboration des châssis cles roses. Fn effet si par sa forme nò̀ recherchée l'image de la rouc appartient sans doute à l'iconographie de l'cutrée nord de la cathédrale, son cmplacement fut choisi en fonction des verrières qui éclairaicut la première travéc de la salle du trésor et non en fonction du portail historié

Malgré sa fonction essentiellement ornementale ct son apparence archaïque, l'exécution de la rose du résor s’inscrit ćgalement pami les innovations du chanticr quant à l'éclairage de l'édificc. Ce problìme constituait unc préoccupation majeure de l'architcoture gothique à cette époque. Le man̂tre du troisième chanticr icntera d'ourir au maximum le mur regroupant plusicurs fençures à chaque travéc. Dans le mur épais du transcpl, clans une forme décloubléc à cause des différents passages supcrposés qui s'ouvrent à l'intérieur puis à l'extéricur, il aménagera 
deux fenêtres surmontées d'un petit trilobc, ajourant le tympan mural sous la section de berceall qui décharge le mur. Dans le mur simple du chocur, les proportions de l'ćlévation ne lui permirent d'ourrir que deux lancetles au cintre très pointu sous un aro doublé par l'arc lormeret de la roûte; unc section centrale restail areugle à la partie supéricure contre ces deux fonctres. Ln troisième trpe de regroupement fut pratiqué à la chapelle épiscopalc du côté sud du transcpt, la dernière sans doute à avoir été conçue (vers 1180$)$. L'édilice de petite dimension ne posatit pas de récl problème de structure: on y regrompa à chaque travéc deux lancettes surmontécs d'un oculus mais le mur entre ces fenêtres et sous le voutain restait bien présent.

Les historicns de l'architecture recomnaissent habituellemont dans cetle dernière formule le modèle qui mène aux fonctres de la nef de Nome-l)ame de Chartres comcues vers $1195^{51}$ (Fig. 16). lin cller. le regroupement v cost scmblable, même si à Charmes l'oculus est agrandi en une rose qui s'inscrit directement sous l'arc lormeret de la voûte. Celte composition aura unc grande répercussion dans le (ćvelop)pement ultéricur d'un nouveau système d'articula- tion de la travéc intégrant les piles, les fonêtres et les voûtes. et où scule la rose allait permettre d'ouvrir complètement le mur. Or ce type d'inscrtion de la rose sous l'arc lormeret de la voûte qui permettait d'ourrir tout le mur entre les piles de division des travées avail ćć pratiqué à deux reprises à Voyon: à la rose octalobéc de la nef et à la rose du irésor.

La rose de la salle du trésor nous fait donc découvrir que le chantice de la cathédrale de Noyon vers $1170-1185$ lut concore plus important pour le développement de la grande fenêtre gothique composéc qu'on ne l'avait crû jusqu’à maintenanı. Non sculement le maîte de (hartres put-il y trouver la composition de la double lancette surmontée d'unc fenctre circulaire, mais il pul également observer a Novon le percement de la rose en fonction de la structure de la voûte.

51 J. Bont. Findh Gothe Architecture of the 12 th and 13 th Cienturies, los Angeles, 1083, p. 235,238 el 230 . A lat (hatpelle épiscopale de Voyour, tout comme a lis cathédrale de Chartes, la dimension des oculi variat selon la largent des travées. Ainsi a Noyon il y avait une neue difference entre les oculi des travees droites et ceux de l'hémicycle.
Figura: ifi. Callédrale de Chartres, fonemes de la nef, vers 1195 (l'hoto: Autcur)

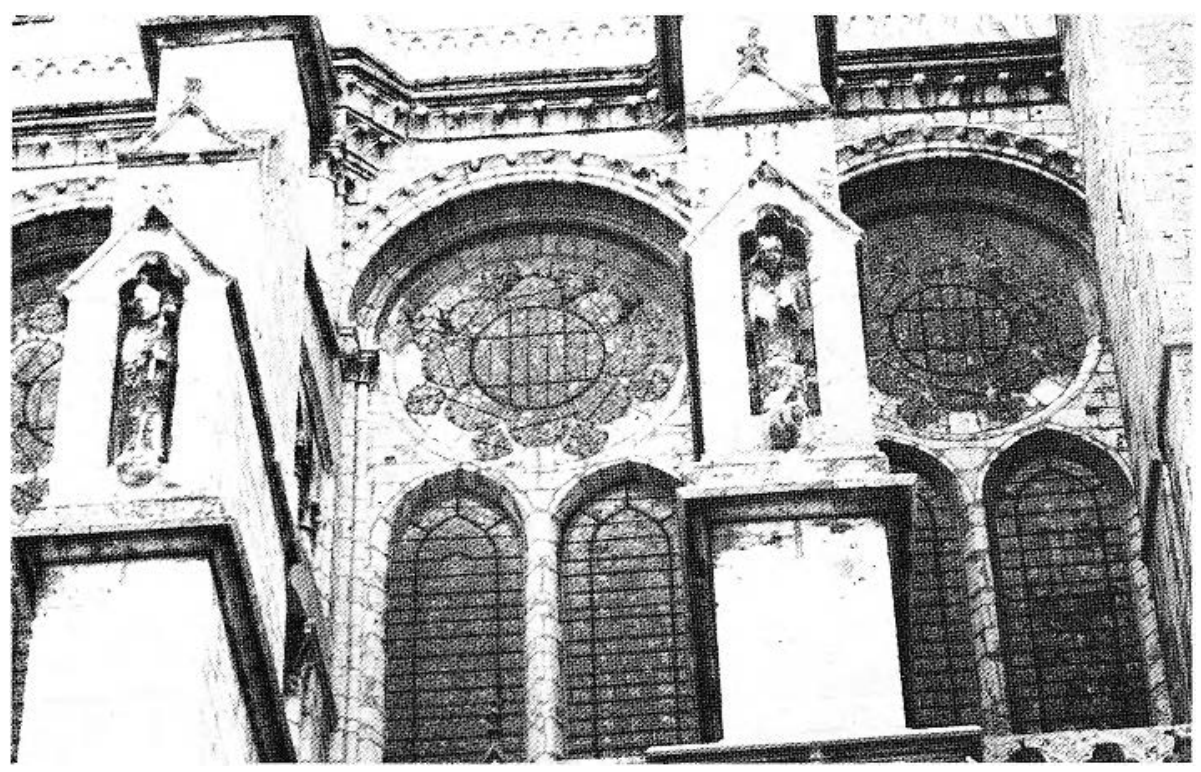

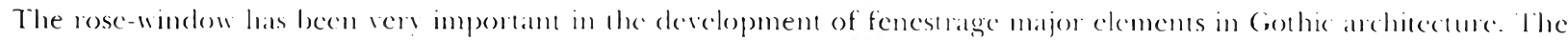

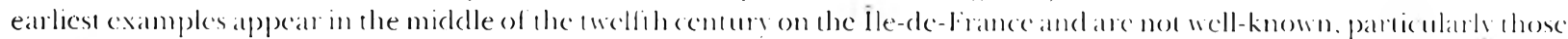

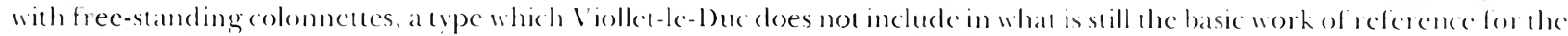
rose-windew. There is a very old window of this wpe preserved in the cathedeal of Noyon. A catreful studs reveals the

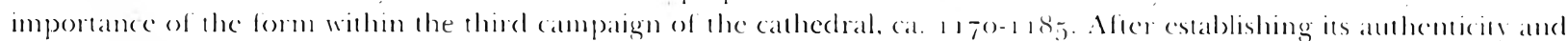

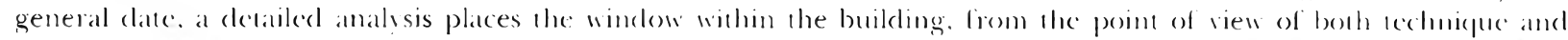

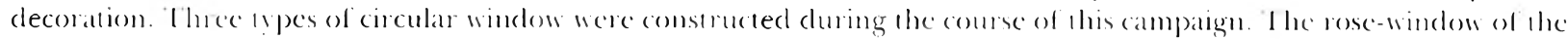

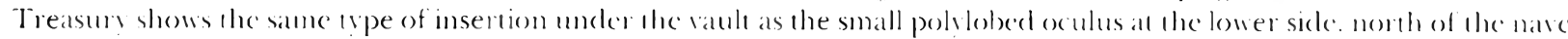




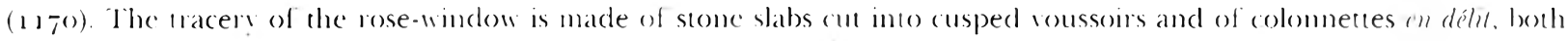

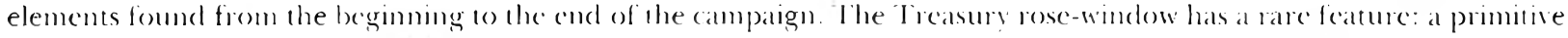
method for securing the frame. The armature is fastened whe interion surface of the curved wedges learing the colonneltes

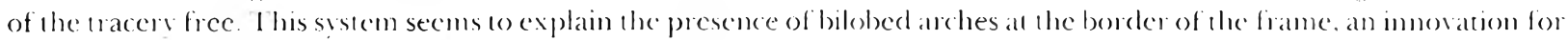
the rose-window wh colonnettes. The decoration of the rose-window is linked wo the secondary decoration of the portal of

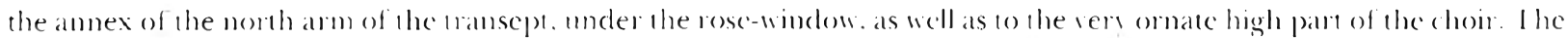

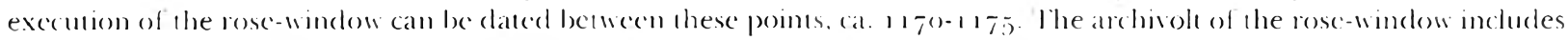

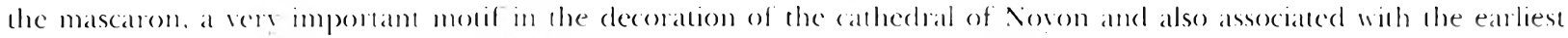
rose-windows (Saint-1)enis. (at. 11.fo). The workshop of Voron is an important center in the development of (ionhic

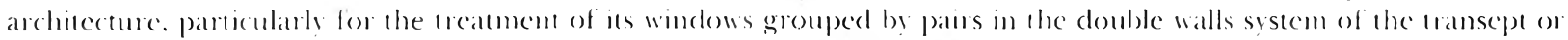
composed with an oculus orer win lancets in the Bishop schapel. This last formula is recognized as the most direct source for the composition of the high windows of the nave of Chatrescathedral. However al (hartres the rose-window hollows out the

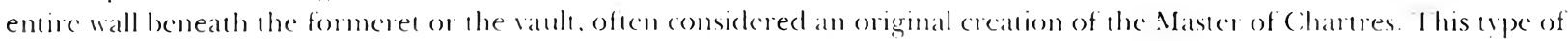

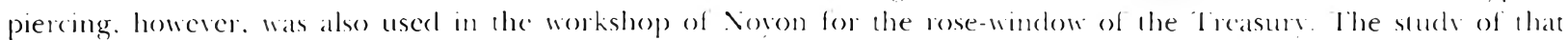
rose-window accentuates, through this fact, the importance of the workshop in the development of the large elaborate gonhic windlow. 